\title{
A Five-Year Study of File-System Metadata
}

\author{
NITIN AGRAWAL \\ University of Wisconsin, Madison \\ and \\ WILLIAM J. BOLOSKY, JOHN R. DOUCEUR, and JACOB R. LORCH \\ Microsoft Research
}

For five years, we collected annual snapshots of file-system metadata from over 60,000 Windows PC file systems in a large corporation. In this article, we use these snapshots to study temporal changes in file size, file age, file-type frequency, directory size, namespace structure, file-system population, storage capacity and consumption, and degree of file modification. We present a generative model that explains the namespace structure and the distribution of directory sizes. We find significant temporal trends relating to the popularity of certain file types, the origin of file content, the way the namespace is used, and the degree of variation among file systems, as well as more pedestrian changes in size and capacities. We give examples of consequent lessons for designers of file systems and related software.

Categories and Subject Descriptors: D.4.3 [Operating Systems]: File Systems ManagementDirectory structures; file organization

General Terms: Measurement, Theory

Additional Key Words and Phrases: File systems, generative model, longitudinal study

\section{ACM Reference Format:}

Agrawal, N., Bolosky, W. J., Douceur, J. R., and Lorch, J. R. 2007. A five-year study of file system metadata. ACM Trans. Storage 3, 3, Article 9 (October 2007), 32 pages. DOI = 10.1145/1288783.1288788 http://doi.acm.org/ 10.1145/1288783.1288788

\section{INTRODUCTION}

Every year from 2000 to 2004, we collected snapshots of metadata from over 10,000 file systems on the Windows desktop computers at Microsoft

This is an expanded version of a paper presented at the 5 th USENIX Conference on File and Storage Technologies (FAST) [Agrawal et al. 2007].

Author's addresses: N. Agrawal, Department of Computer Science, University of WisconsinMadison, 7361 Computer Sciences and Statistics, 1210 W. Dayton St., Madison, WI 53706; email: nitina@cs.wisc.edu; W. J. Bolosky, J. R. Douceur, J. R. Lorch, Microsoft Research, One Microsoft Way, Redmond, WA 980927; email: \{bolosky, johndo, lorch\}@microsoft.com

Permission to make digital or hard copies of part or all of this work for personal or classroom use is granted without fee provided that copies are not made or distributed for profit or direct commercial advantage and that copies show this notice on the first page or initial screen of a display along with the full citation. Copyrights for components of this work owned by others than ACM must be honored. Abstracting with credit is permitted. To copy otherwise, to republish, to post on servers, to redistribute to lists, or to use any component of this work in other works requires prior specific permission and/or a fee. Permissions may be requested from Publications Dept., ACM, Inc., 2 Penn Plaza, Suite 701, New York, NY 10121-0701 USA, fax +1 (212) 869-0481, or permission@acm.org. (C) 2007 ACM 1553-3077/2007/10-ART9 \$5.00 DOI = 10.1145/1288783.1288788 http://doi.acm.org/ $10.1145 / 1288783.1288788$ 
Corporation. We gathered this data by mass-emailing a scanning program to Microsoft's employees, and we had a $22 \%$ participation rate every year. Our resulting datasets contain metadata from 63,398 distinct file systems, 6457 of which provided snapshots in multiple years.

This project was a longitudinal extension of an earlier study we performed in 1998 [Douceur and Bolosky 1999], which was an order of magnitude larger than any prior study of file-system metadata. Our earlier study involved a single capture of file-system metadata, and focused on lateral variation among file systems at a moment in time. By contrast, the present study focuses on longitudinal changes in file systems over a five-year time span.

In particular, we study temporal changes in the size, age, and type frequency of files; the size of directories; the structure of the file-system namespace; and various characteristics of file systems, including file and directory population, storage capacity, storage consumption, and degree of file modification.

The contributions of this work are threefold. First, we contribute the collected dataset. This is the largest set of file-system metadata ever collected, and it spans the longest time period of any sizeable metadata collection. It is available at the Storage Networking Industry Association's IOTTA repository, at http://iotta.snia.org/traces/tracesStaticSnapshot/.

Second, we contribute all of our research observations, including the following.

- The space used in file systems has increased over the course of our study, not only because mean file size has increased (from $108 \mathrm{~KB}$ to $189 \mathrm{~KB}$ ), but also because the number of files has increased (from $30 \mathrm{~K}$ to $90 \mathrm{~K}$ ).

-Eight file name extensions account for over $35 \%$ of files, and nine file name extensions account for over $35 \%$ of the bytes in files. The same sets of extensions have remained popular for many years.

- The fraction of file-system content created or modified locally has decreased over time. In the first year of our study, the median file-system had 30\% of its files created or modified locally, and four years later this percentage was $22 \%$.

-Directory size distribution has not notably changed over the years of our study. In each year, directories have had very few subdirectories and a modest number of entries. $90 \%$ of them have had two or fewer subdirectories, and the same percentage has had 20 or fewer total entries.

- The fraction of file-system storage residing in the namespace subtree meant for user documents and settings has increased in every year of our study, starting at $7 \%$ and rising to $15 \%$. The fraction residing in the subtree meant for system files has also risen over the course of our study, from $2 \%$ to $11 \%$.

-File system capacity has increased dramatically during our study, with median capacity rising from $5 \mathrm{~GB}$ to $40 \mathrm{~GB}$. One might expect this to cause drastic reductions in file-system fullness, but instead this reduction has been modest. Median fullness has only decreased from $47 \%$ to $42 \%$.

- Over the course of a single year, $80 \%$ of file systems become fuller and $18 \%$ become less full. 
Third, we contribute a generative, probabilistic model for how directory trees are created. Our model explains the distribution of directories by depth in the namespace tree, and also explains the distribution of the count of subdirectories per directory. This is the first generative model that characterizes the process by which file-system namespaces are constructed.

Section 2 describes the methodology of our data collection, analysis, and presentation. Sections 3, 4, and 5 present our findings on, respectively, files, directories, and space usage. Section 6 surveys related work, and Section 7 summarizes and concludes.

\section{METHODOLOGY}

This section describes the methodology we applied to collecting, analyzing, and presenting the data. The collection was performed via voluntary participation of each machine's user, recognizing that the voluntary approach could produce selection bias. Analyses include analytical modeling of certain distributions of interest, generative modeling of one particular distribution, and year-to-year comparisons for those file systems for which we have snapshots in multiple years.

\subsection{Data Collection}

We developed a simple program that traverses the directory tree of each local, fixed-disk file-system mounted on a computer. The program records a snapshot of all metadata associated with each file or directory, including hidden files and directories. This metadata includes name, size, timestamps, and attributes. The program also records the parent-child relationships of nodes in the namespace tree, as well as some system configuration information. The program records file names in an encrypted form. We wrote automated tools that decrypt the file names for computing aggregate statistics, but for privacy reasons we do not look at the decrypted file names directly, which places some limits on our analyses. In postprocessing, we remove metadata relating to the system paging file because this is part of the virtual memory system rather than the file-system.

In the autumn of every year from 2000 to 2004, we distributed the scanning program via email to a large subset of the employees of Microsoft, with a request for the recipients to run the program on their desktop machines. As an incentive to participate, we held a lottery in which each scanned machine counted as an entry, with a single prize of a night's stay at a nearby resort hotel. The specific subset of people we were permitted to poll varied from year to year based on a number of factors; however, despite variations in user population and in other distribution particulars, we observed a $22 \%$ participation rate every year.

We scanned desktops rather than servers because at Microsoft, files are typically stored on individual desktops rather than centralized servers. We collected the data via voluntary participation rather than random selection because the company only permitted the former approach; note that this voluntary approach may have produced selection bias. 
Table I. Properties of Each Year's Dataset

\begin{tabular}{|clccc|}
\hline Year & \multicolumn{1}{c}{ Period } & Users & Machs & FSs \\
\hline \hline 2000 & 13 Sep-29 Sep & 5396 & 6051 & 11,654 \\
\hline 2001 & 8 Oct-2 Nov & 7539 & 9363 & 16,022 \\
\hline 2002 & 30 Sep-1 Nov & 7158 & 9091 & 15,011 \\
\hline 2003 & 13 Oct-14 Nov & 7436 & 9262 & 14,633 \\
\hline 2004 & 5 Oct-12 Nov & 7180 & 8729 & 13,505 \\
\hline
\end{tabular}

Table II. File System Types in Datasets

\begin{tabular}{|rrrrcr|}
\hline Year & NTFS & FAT32 & FAT & Other & Total \\
\hline \hline 2000 & 7,015 & 2,696 & 1,943 & 0 & 11,654 \\
\hline 2001 & 11,791 & 3,314 & 915 & 2 & 16,022 \\
\hline 2002 & 12,302 & 2,280 & 429 & 0 & 15,011 \\
\hline 2003 & 12,853 & 1,478 & 302 & 0 & 14,633 \\
\hline 2004 & 12,364 & 876 & 264 & 1 & 13,505 \\
\hline Total & 56,325 & 10,644 & 3,853 & 3 & 70,825 \\
\hline
\end{tabular}

\subsection{Data Properties}

Table I itemizes some of the properties of each year's data collection. The primary collection period ran between the listed start and end dates, which mark the beginning of our emailing requests and the last eligible day for the lottery. Some snapshots continued to trickle in after the primary collection period; we used these in our analyses as well.

Table II itemizes the breakdown of each year's snapshots according to filesystem type. Specifically, $80 \%$ of our snapshots came from NTFS [Solomon 1998], the main file-system for operating systems in the Windows NT family; $5 \%$ from FAT [Mitchell 1997], a 16-bit file-system dating from DOS; and 15\% from FAT32 [Mitchell 1997], a 32-bit upgrade of FAT developed for Windows 95.

For some analyses, we needed a way to establish whether two file-system snapshots from different years refer to the same file-system. "Sameness" is not actually a well-formed notion; for example, it is not clear whether a file-system is still the same after its volume is extended. We defined two snapshots as refering to the same file-system if and only if they have the same user name, computer name, volume ID, drive letter, and total space. The need for some of these conditions was not obvious at first. For example, we added drive letter because some drives on some machines are multiply mapped, and we added total space so that a volume set would not be considered the same if a new volume were added to the set.

Based on this definition, Table III shows the number of snapshots for which we have consecutive-year information. Each row indicates, for the indicated year, how many file systems are represented in the dataset captured 1 to 4 years later. The counts fall off quickly: Of the 57K snapshots in the 2000-2003 datasets, only 6500 have corresponding snapshots one year later, and only 1300 have corresponding snapshots two years later. Because we have so few matching snapshot pairs separated by three or more years, we do not use these pairs in our year-to-year comparisons.

ACM Transactions on Storage, Vol. 3, No. 3, Article 9, Publication date: October 2007. 
Table III. Number of File Systems for Which We Have Snapshots

\begin{tabular}{|lccccc|}
\hline Start & $\mathbf{1}$ & $\mathbf{2}$ & $\mathbf{3}$ & $\mathbf{4}$ & $\mathbf{5}$ \\
\hline \hline 2000 & 11,654 & 950 & 234 & 63 & 18 \\
\hline 2001 & 16,022 & 1,833 & 498 & 144 & - \\
\hline 2002 & 15,011 & 1,852 & 588 & - & - \\
\hline 2003 & 14,633 & 1,901 & - & - & - \\
\hline 2004 & 13,505 & - & - & - & - \\
\hline Total & 70,825 & 6,536 & 1,320 & 207 & 18 \\
\hline
\end{tabular}

These snapshots were taken in the $n$ consecutive years starting with each year. For instance, there are 1,852 file systems for which we have snapshots from both 2002 and 2003.

\subsection{Data Presentation}

Many of our graphs have horizontal axes that span a large range of nonnegative numbers. To represent these ranges compactly, we use a logarithmic scale for non-zero values, but we also include an abscissa for the zero value, even though zero does not strictly belong on a logarithmic scale.

We plot most histograms with line graphs rather than bar graphs because with five or more datasets on a single plot, bar graphs can become difficult to read. For each bin in the histogram, we plot a point $(x, y)$, where $x$ is the midpoint of bin and $y$ is the size of bin. We use the geometric midpoint when the $x$ axis uses a logarithmic scale. We often plot unnormalized histograms rather than probability density functions (PDFs) for two reasons: First, the graphs expose more data if we do not normalize them. Second, because the count of files and directories per file-system has grown substantially over time, not normalizing allows us to plot multiple years' curves on the same chart without overlapping to the point of unreadability.

Whenever we use the prefix $\mathrm{K}$, as in $\mathrm{KB}$, we mean $2^{10}$. Similarly, we use M for $2^{20}$ and $\mathrm{G}$ for $2^{30}$.

Although our analytical models are fit to cumulative distributions, we plot them on histograms so as to emphasize any discrepancies between the empirical data and analytical functions.

\subsection{Modeling}

We have developed analytical models for certain distributions of interest. These models are mostly standard distributions whose parameters we determined by curve fitting. An exception is a generative model that characterizes directories by their depth in the namespace tree and by their count of subdirectories.

To develop analytical approximations of empirical data, we select a model that is compatible with both the general shape of the observed distribution and our intuition about the underlying phenomena. We select fitting parameters that minimize the mean square differences between the cumulative curves of the analytical distribution and the observed frequency. We quantify the fit according to the maximum displacement of the cumulative curves (MDCC), which is the greatest absolute vertical separation between the cumulative measured 
frequency curve and the cumulative analytical frequency curve. At a 0.01 level of significance, all of our continuous approximations fail the KolmogorovSmirnov test [Knuth 1981], and all of our discrete approximations fail the chisquare test [Freund 1992]. Therefore, we do not claim to have found governing distributions for our observations. We claim only to have developed graphical approximations whose utility is that they provide a highly compact, if not wholly accurate, representation of the empirical data.

\subsection{Data Analysis}

We believe that analysis of longitudinal file-system data is of interest to many sets of people with diverse concerns about file-system usage. For instance, it could benefit the following persons.

—developers of file systems, including desktop, server, and distributed file systems;

—storage area network designers;

- developers of file-system utilities, such as backup, antivirus, content indexing, encryption, and disk space usage visualization;

- storage capacity planners;

—disk manufacturers, especially those using gray-box techniques to enable visibility into the file-system at the disk level [Arpaci-Dusseau and ArpaciDusseau 2001]; and

-multitier storage system developers.

In each subsection, after discussing our findings and what we consider to be the most interesting summaries of these findings, we will present some examples of interesting implications for the people just enumerated.

\subsection{Limitations}

All our data comes from a relatively homogenous sample of machines: Microsoft desktops running Windows. Since past studies [Roselli et al. 2000; Vogels 1999] have shown that file-system characteristics can vary from one environment to another, they may not be applicable to substantially different environments. For instance, they are likely not applicable to file-system server workloads, and it is unclear to what extent they can be generalized to non-Windows operating systems. It may also be that artifacts of Microsoft policy, such as specific software distributions that are common or disallowed, may yield results that would not apply to other workloads.

\section{FILES}

\subsection{File Count per File System}

Figure 1 plots cumulative distribution functions (CDFs) of file systems by count of files. The count of files per file-system has increased steadily over our fiveyear sample period: The arithmetic mean has grown from $30 \mathrm{~K}$ to $90 \mathrm{~K}$ files and the median has grown from $18 \mathrm{~K}$ to $52 \mathrm{~K}$ files. 


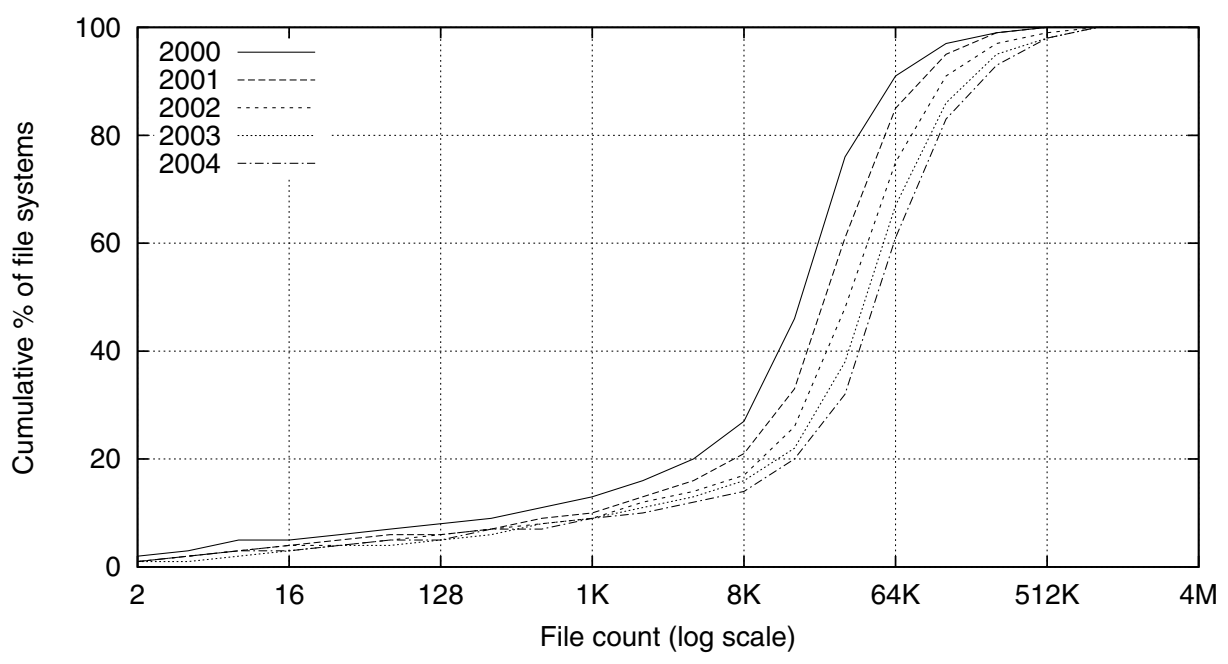

Fig. 1. CDFs of file systems by file count.

The count of files per file-system is going up from year to year, and, as we will discuss in Section 4.1, the same holds for directories. Thus, file-system designers should ensure that their metadata tables scale to large file counts. Additionally, we can expect file-system scans that examine data proportional to the number of files and/or directories to take progressively longer. Examples of such scans include virus scans and metadata integrity checks following block corruption. Thus, it will become increasingly useful to perform these checks efficiently, perhaps by scanning in an order that minimizes movement of the disk arm.

\subsection{File Size}

This section describes our findings regarding file size. We report the size of actual content, ignoring the effects of internal fragmentation, file metadata, and any other overhead. We observe that the overall file size distribution has changed slightly over the five years of our study. By contrast, the majority of stored bytes are found in increasingly larger files. Moreover, the latter distribution increasingly exhibits a double mode, due mainly to database and blob (binary large object) files.

Figure 2 plots histograms of files by size and Figure 3 the corresponding CDFs. We see that the absolute count of files per file-system has grown significantly over time, but the general shape of the distribution has not changed significantly. Although it is not visible on the graph, the arithmetic mean file size has grown by $75 \%$ from $108 \mathrm{~KB}$ to $189 \mathrm{~KB}$. In each year, $1-1.5 \%$ of files have a size of 0 .

The growth in mean file size from $108 \mathrm{~KB}$ to $189 \mathrm{~KB}$ over four years suggests that this metric grows roughly $15 \%$ per year. Another way to estimate this growth rate is to compare our 2000 result to the 1981 result of $13.4 \mathrm{~KB}$ obtained by Satyanarayanan [1981]. This comparison estimates the annual growth rate 


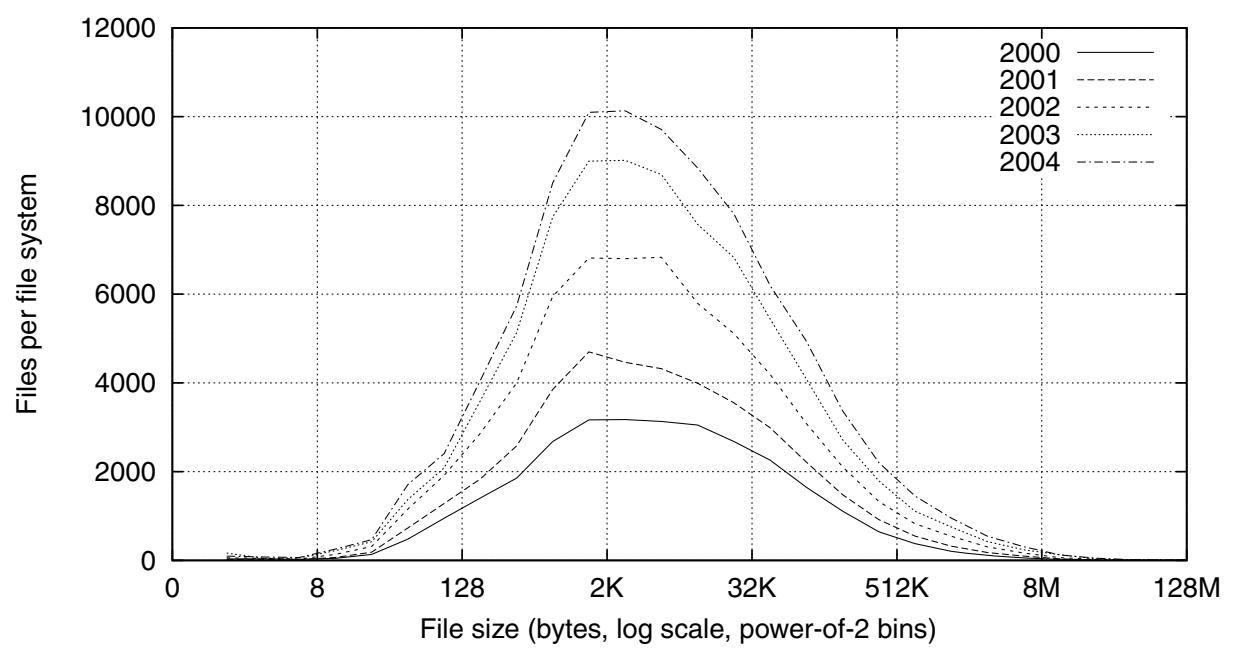

Fig. 2. Histograms of files by size.

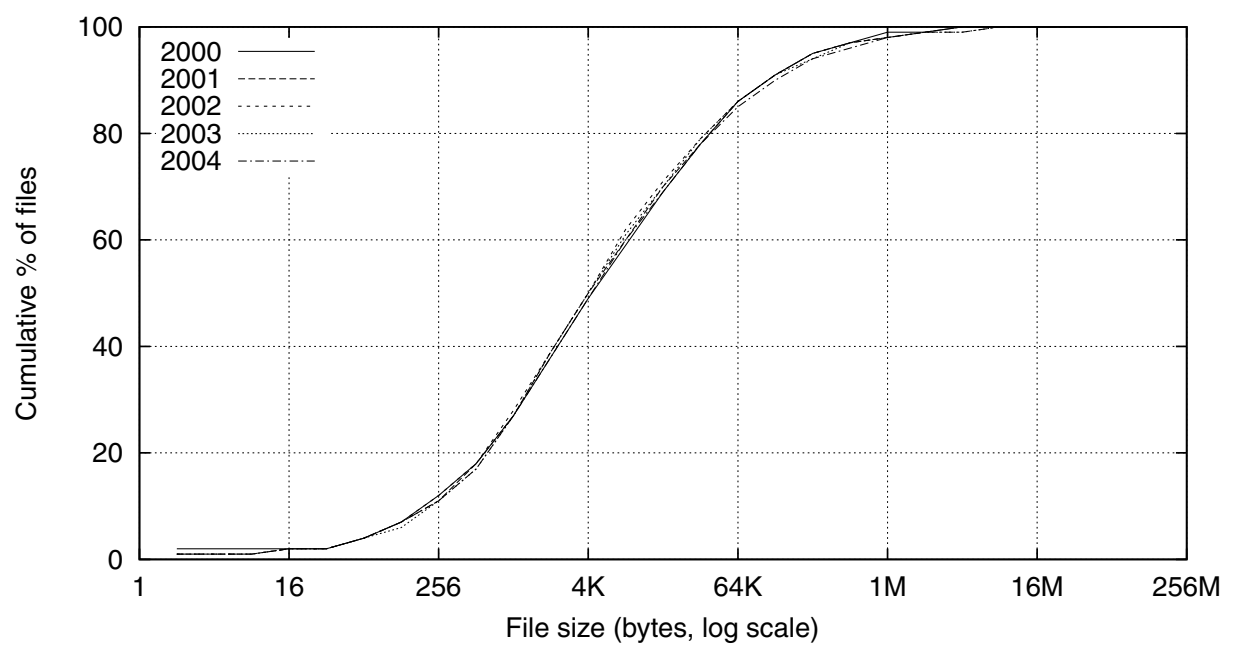

Fig. 3. CDFs of files by size.

as $12 \%$. Note that this latter estimate is somewhat flawed, since it compares file sizes from two rather different environments.

Figure 4 plots histograms of bytes by containing file size, alternately described as histograms of files weighted by file size. Figure 5 plots the CDFs of these distributions. We observe that the distribution of file size has shifted to the right over time, with the median weighted file size increasing from $3 \mathrm{MB}$ to $9 \mathrm{MB}$. Also, the distribution exhibits a double mode that has become progressively more pronounced. The corresponding distribution in our 1998 study did not show a true second mode, but did show an inflection point around $64 \mathrm{MB}$, which is near the local minimum in Figure 4. 


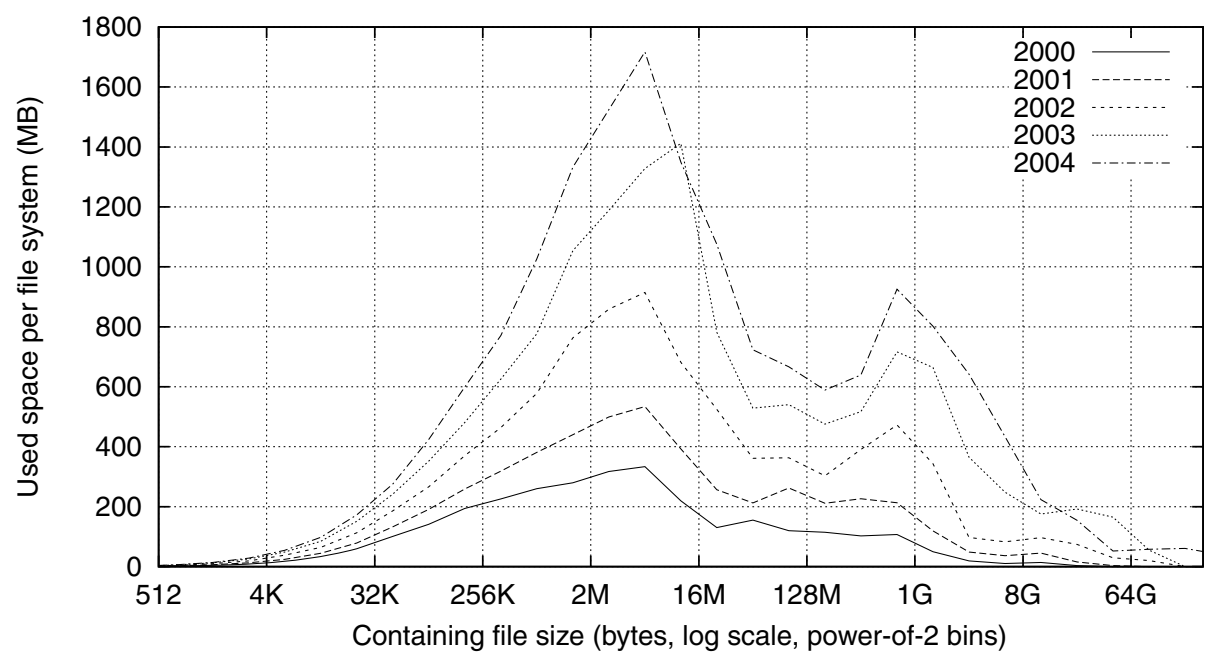

Fig. 4. Histograms of bytes by containing file size.

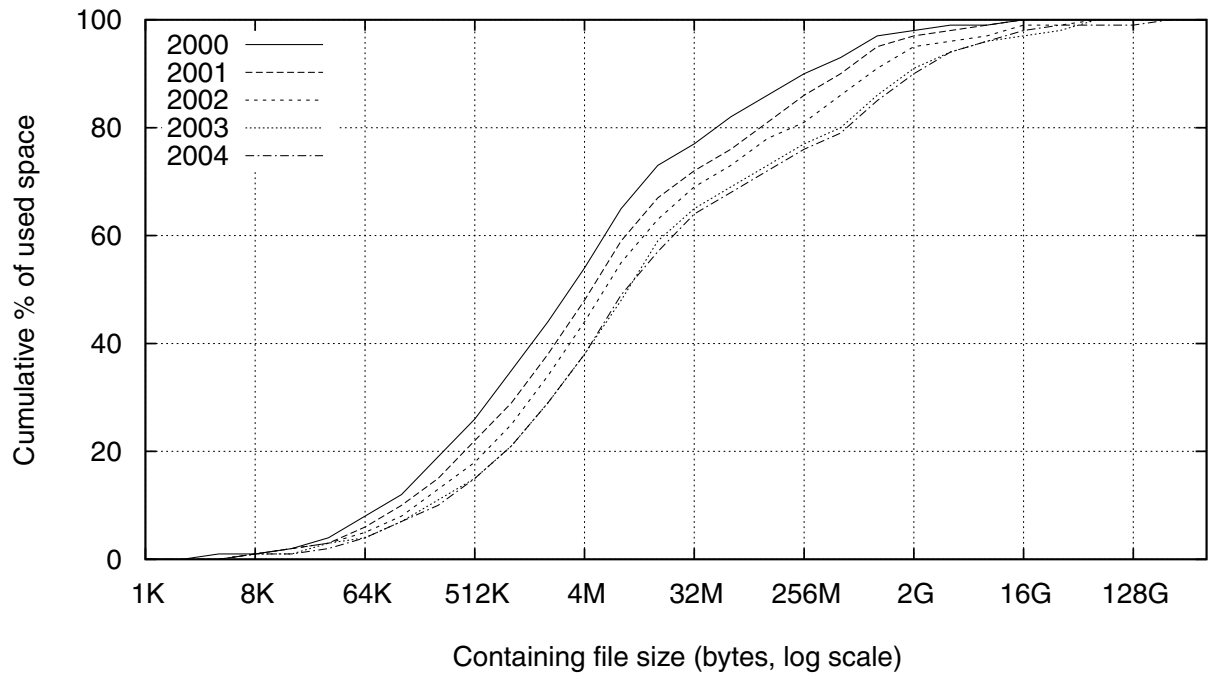

Fig. 5. CDFs of bytes by containing file size.

To study this second peak, we broke out several categories of files according to file-name extension. Figure 6 replots the 2004 data from Figure 4 as a stacked bar chart, with the contributions of video, database, and blob files indicated. We see that most of the bytes in large files are in video, database, and blob files, and that most of the video, database, and blob bytes are in large files.

Our finding that different types of files have different size distributions echoes the findings of other studies. Over two decades ago, Satyanarayanan [1981] found this to be the case on a shared file server in an academic environment. In 2001, Evans and Kuenning also noted this phenomenon in their analysis of 22 machines running various operating systems at Harvey Mudd 


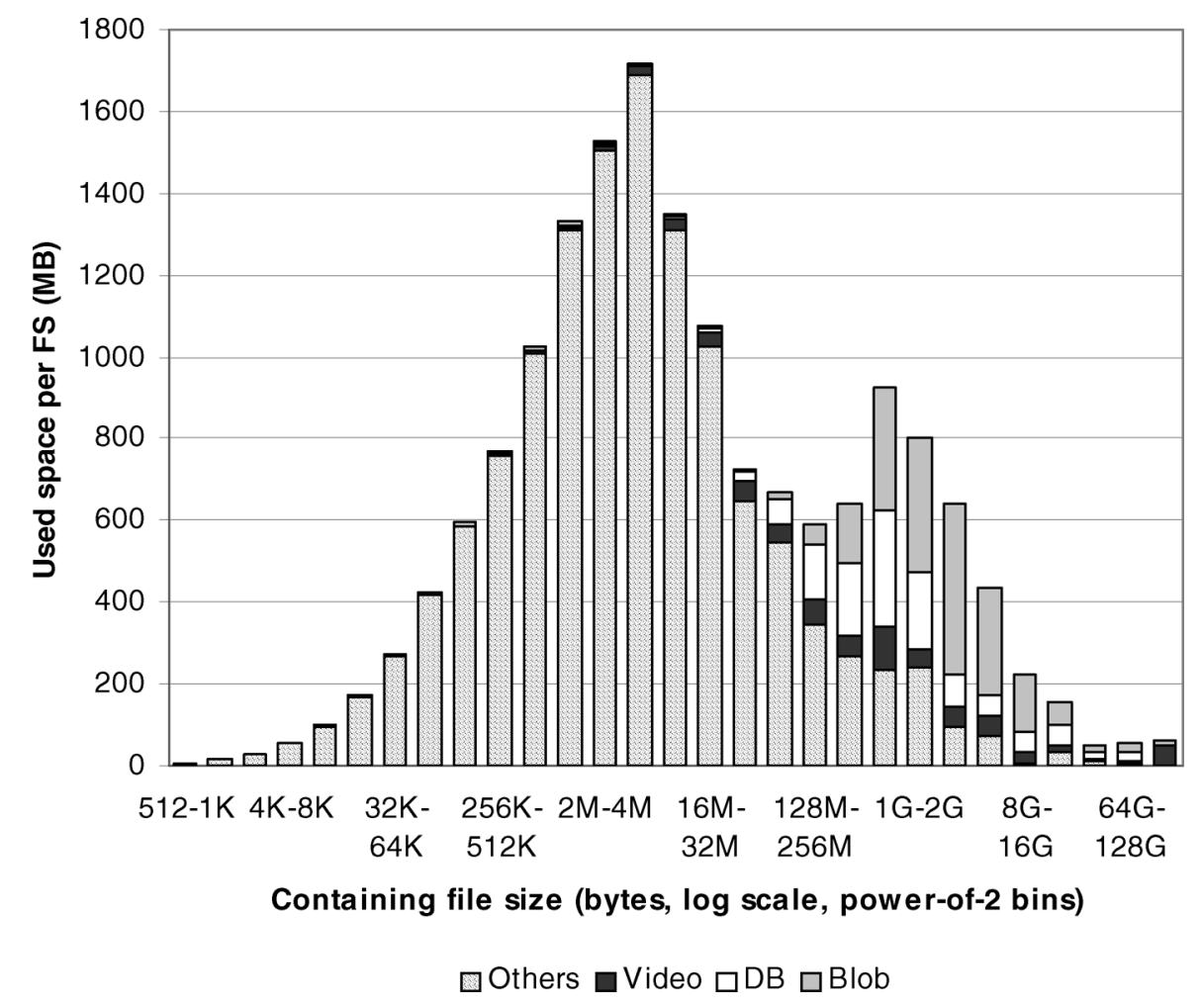

Fig. 6. Contribution of file types to Figure 4 (2004). Video means files with extensions avi, dps, mpeg, mpg, vob, or wmv; $D B$ means files with extensions ldf, mad, mdf, ndf, ost, or pst; and Blob means files named hiberfil.sys and files with extensions bak, bkf, bkp, dmp, gho, iso, pqi, rbf, or vhd.

College and Marine Biological Laboratories [Evans and Kuenning 2002]. The fact that this finding is consistent across various environments and times suggests that it is fundamental.

There are several implications of the fact that a large number of small files account for a small fraction of disk usage. First, it may not take much space to colocate many of these files with their metadata. This may be a reasonable way to reduce the disk seek time needed to access these files. Second, a file-system that colocates several files in a single block, like ReiserFS [Reiser 2006], will have many opportunities to do so. This will save substantial space by eliminating internal fragmentation, especially if a large block size is used to improve performance. Third, designers of disk usage visualization utilities may want to show not only directories, but also the names of certain large files.

\subsection{File Age}

This subsection describes our findings regarding file age. Because file timestamps can be modified by application programs [Microsoft 2006], our conclusions should be regarded cautiously. 


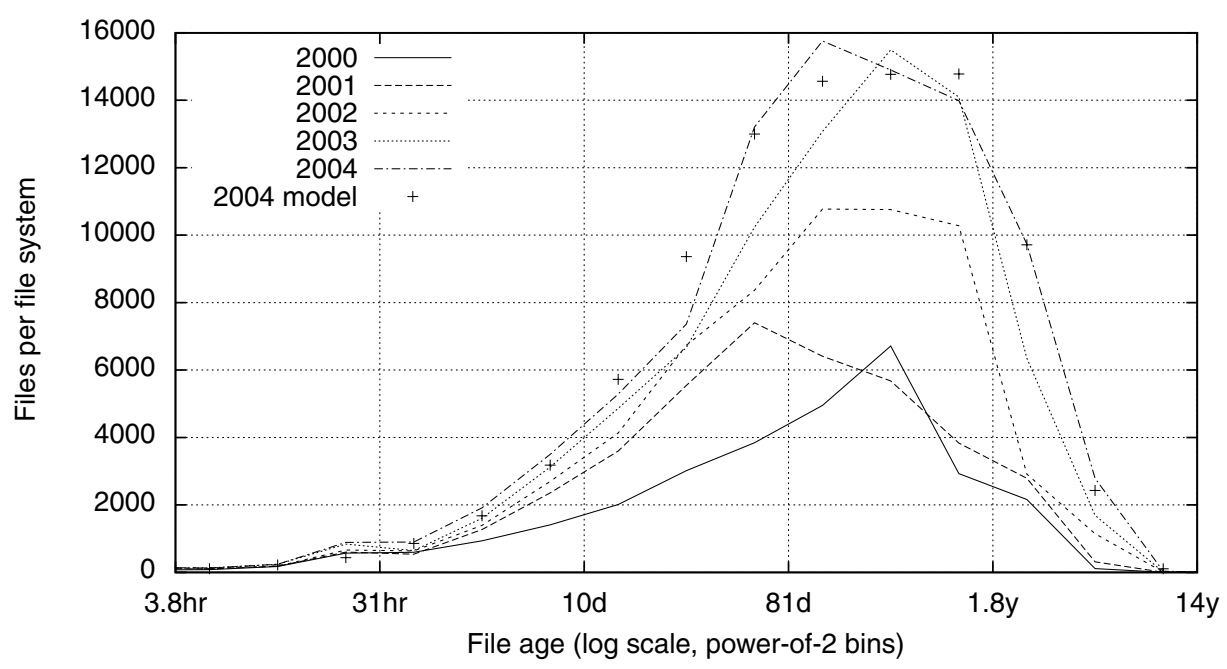

Fig. 7. Histograms of files by age.

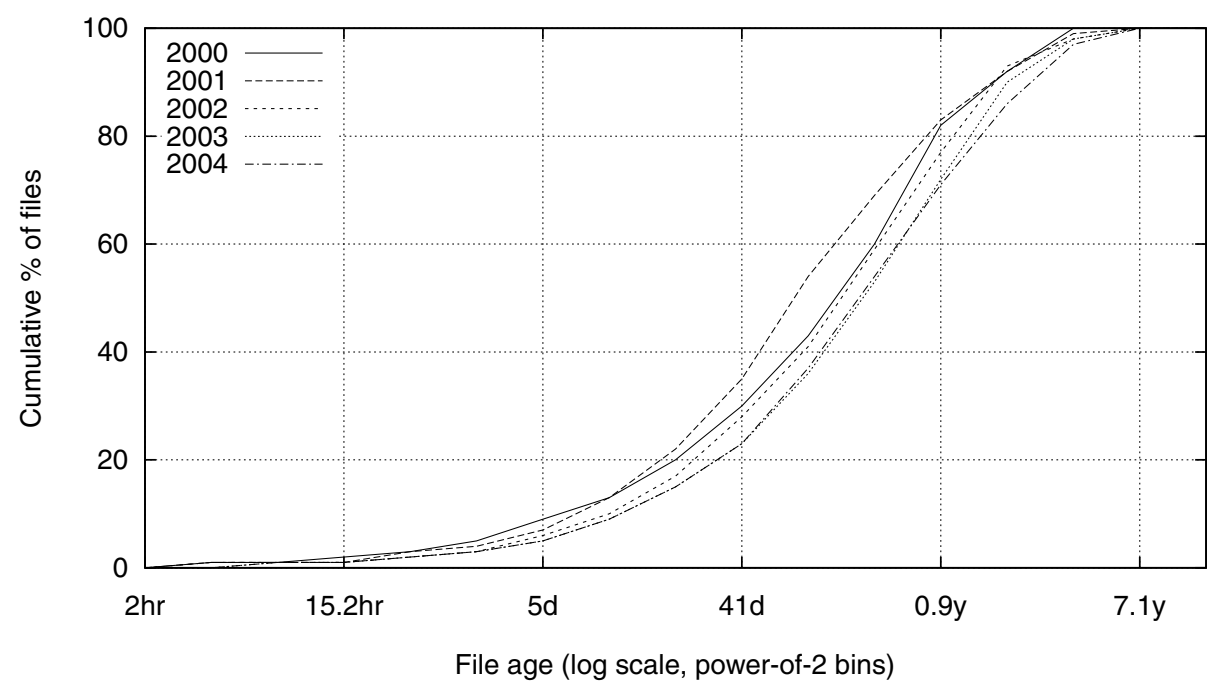

Fig. 8. CDFs of files by age.

Figure 7 plots histograms of files by age, calculated as the elapsed time since the file was created or last modified relative to the time of the snapshot. Figure 8 shows the CDFs of this same data. The median file age ranges between 80 and 160 days across datasets, with no clear trend over time.

The age distribution can be approximated with a two-stage hyperexponential distribution, consistent with our findings in 1998. Figure 7 plots a hyperexponential for the 2004 dataset $\alpha_{0}=0.65, \mu_{0}=2^{25.1} \mathrm{sec}, \alpha_{1}=0.35, \mu_{1}=2^{22.3}$ sec. The MDCC is $2 \%$.

The distribution of file age is not memoryless, so the age of a file is useful in predicting its remaining lifetime. So, systems such as archival backup can use 
Table IV. Typical Usage of Popular File Extensions

\begin{tabular}{|l|l|}
\hline Extension & Typical Usage \\
\hline \hline cpp & C++ source code \\
\hline dll & Dynamic link library \\
\hline exe & Executable \\
\hline gif & Image in Graphic Interchange Format \\
\hline h & Source code header \\
\hline htm & File in hypertext markup language \\
\hline jpg & Image in JPEG format \\
\hline lib & Code library \\
\hline mp3 & Music file in MPEG Layer III format \\
\hline pch & Precompiled header \\
\hline pdb & Source symbols for debugging \\
\hline pst & Outlook personal folder \\
\hline txt & Text \\
\hline vhd & Virtual hard drive for virtual machine \\
\hline wma & Windows Media Audio \\
\hline
\end{tabular}

this distribution to make predictions of how much longer a file will be needed based on how old it is. Since the distribution of file age has not appreciably changed across the years, we can expect that a prediction algorithm developed today based on the latest distribution will apply for several years to come.

\subsection{File-Name Extensions}

This subsection describes our findings regarding popular file types as determined by file-name extension. Although the top few extensions have not changed dramatically over our five-year sample period, there has been some alteration, reflecting a decline in the relative prevalence of web content and an increase in use of virtual machines. The top few extensions account for nearly half of all files and bytes in file systems.

In old DOS systems with 8.3-style file names, the extension was the zero to three characters following the single dot in the file name. Although Windows systems allow file names of nearly arbitrary length and containing multiple dots, many applications continue to indicate their file types by means of extensions. For our analyses, we define an extension as the five or fewer characters following the last dot in a file name. If a name has no dots or more than five characters after the last dot, we consider that name to have no extension, which we represent with the symbol $\varnothing$. As a special case, if a file name ends in .gz, .bz2, or .Z, then we ignore that suffix when determining extension. We do this because these are types of compressed files wherein the actual content type is indicated by the characters prior to the compression extension. To understand the typical usage of the file extensions we discuss in this section, see Table IV.

Figure 9 plots, for the nine most popular extensions in terms of file count, the fraction of files with that extension. The fractions are plotted longitudinally over our five-year sample period. The most notable thing we observe is that these extensions' popularity is relatively stable; the top five extensions have remained 50 for this entire time. However, the relative popularity of gif and htm files has 


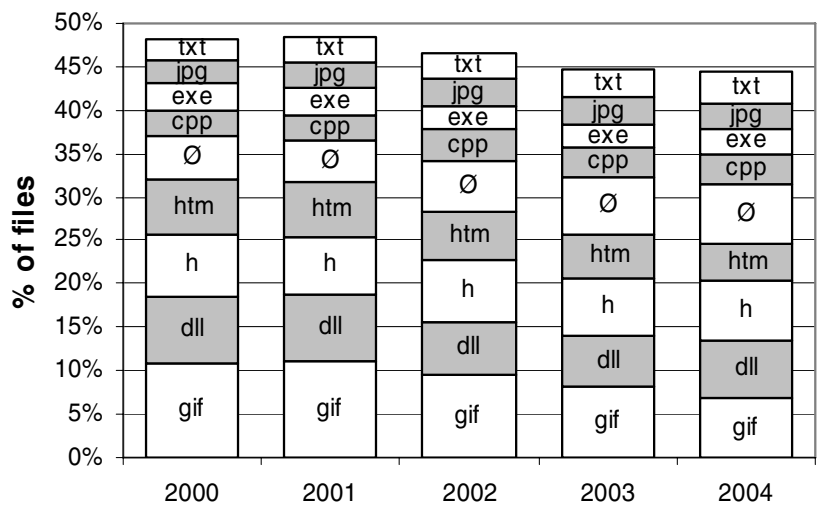

Fig. 9. Fraction of files with popular extensions.

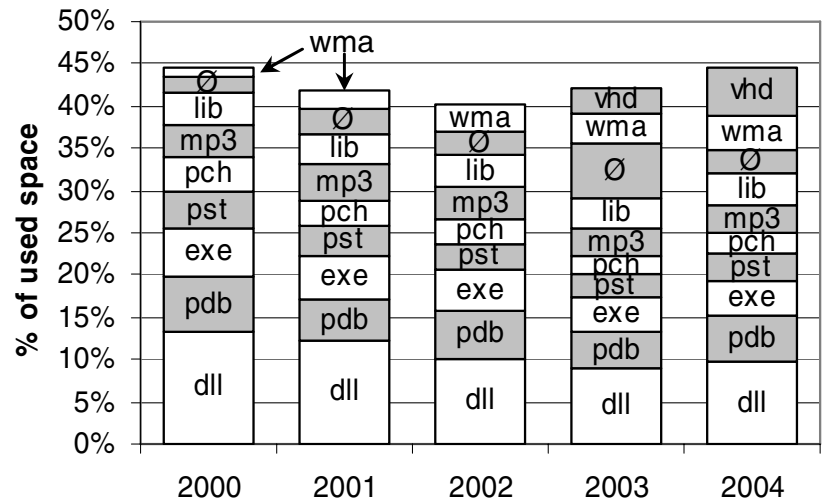

Fig. 10. Fraction of bytes in files with popular extensions.

gone down steadily since 2001, suggesting a decline in the popularity of web content relative to other ways to fill one's file-system.

Figure 10 plots, for the ten most popular extensions in terms of summed file size, the fraction of file bytes residing in files with that extension. Across all years, dynamic link libraries (dll files) contain more bytes than any other file type. Extension vhd, which is used for virtual hard drives, is consuming a rapidly increasing fraction of file-system space, suggesting that virtual machine use is increasing. The null extension exhibits a notable anomaly in 2003 , but we cannot investigate the cause without decrypting the file names in our datasets, which would violate our privacy policy.

Since files with the same extension have similar properties and requirements, some file-system management policies can be improved by including special-case treatment for particular extensions. Such special-case treatment can be built into the file-system or autonomically and dynamically learned [Mesnier et al. 2004]. Since nearly half the files, as well as nearly half the bytes, belong to files with a few popular extensions, developing such specialcase treatment for only a few particular extensions can optimize performance 
9:14 $\quad$ N. Agrawal et al.

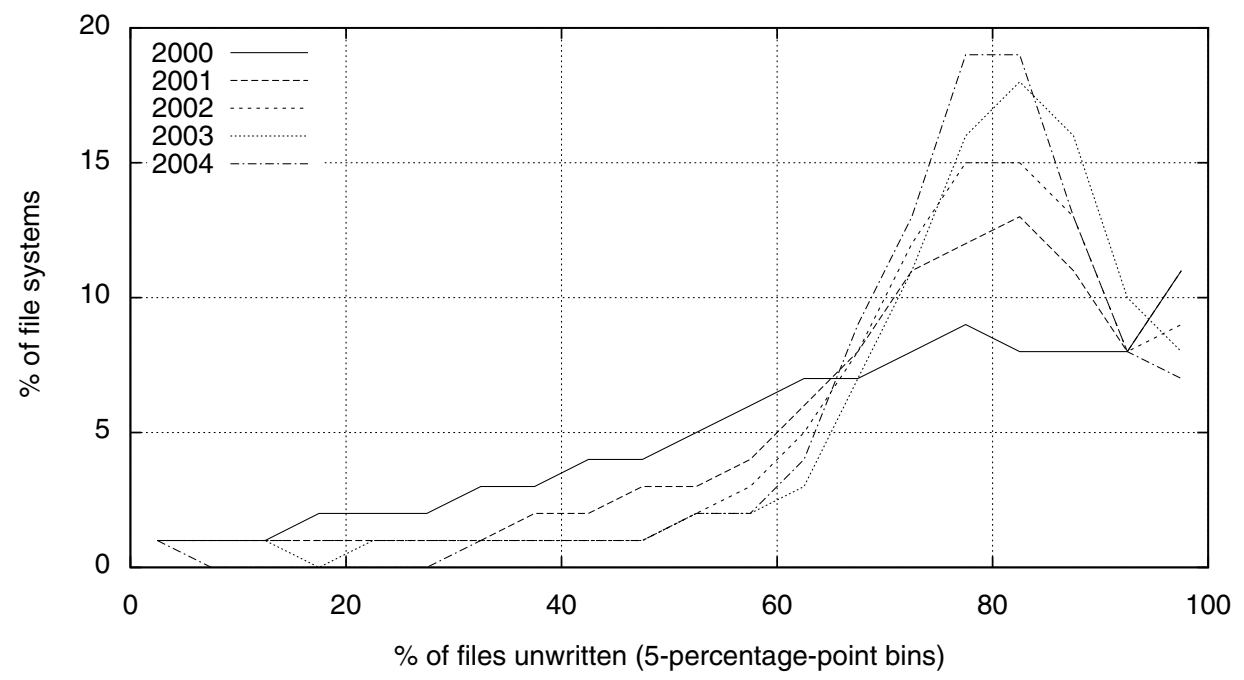

Fig. 11. Histograms of file systems by percentage of files unwritten.

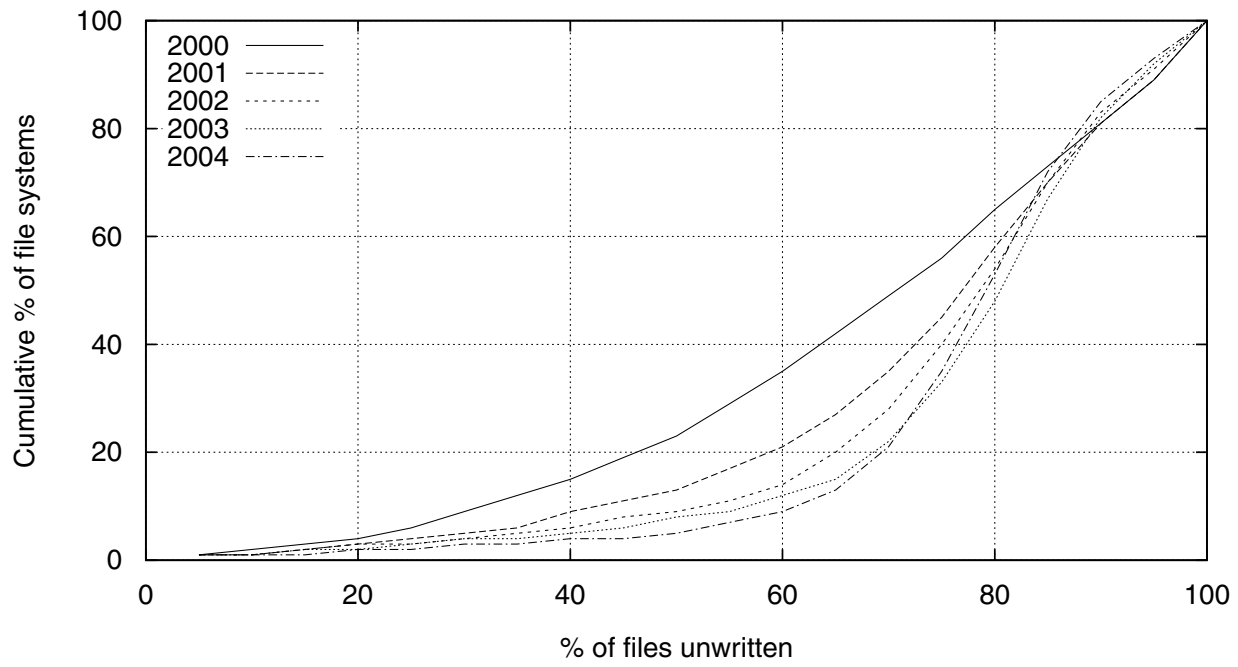

Fig. 12. CDFs of file systems by percentage of files unwritten.

for a large fraction of the file-system. Furthermore, since the same extensions continue to be popular year after year, one can develop special-case treatments for today's popular extensions and expect that they will still be useful years from now.

\subsection{Unwritten Files}

Figures 11 and 12 plot the histograms and CDFs, respectively, of file systems by percentage of files that have not been written since being copied onto the file-system. We identify such files as ones whose modification timestamps are earlier than their creation timestamps, since the creation timestamp of a copied 


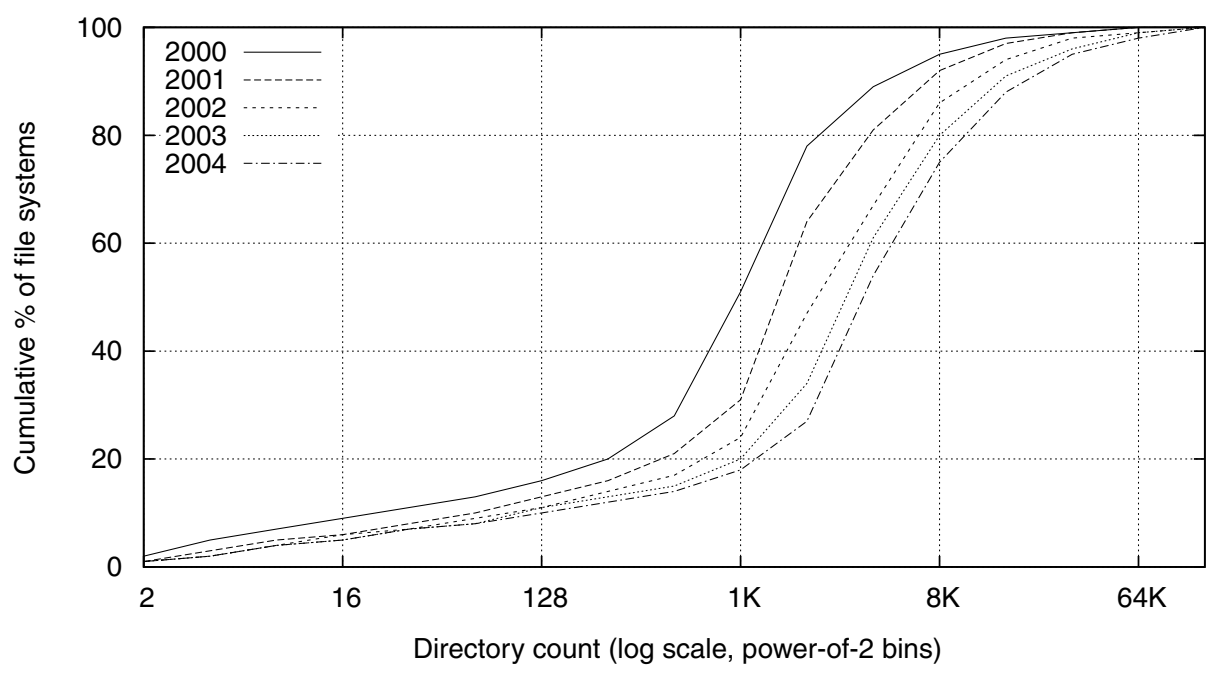

Fig. 13. CDFs of file systems by directory count.

file is set to the time at which the copy was made, but its modification timestamp is copied from the original file. Over our sample period, the arithmetic mean of the percentage of locally unwritten files has grown from $66 \%$ to $76 \%$, and the median has grown from $70 \%$ to $78 \%$. This suggests that users locally contribute to a decreasing fraction of their systems' content. This may in part be due to the increasing amount of total content over time.

Since more and more files are being copied across file systems rather than generated locally, we can expect identifying and coalescing identical copies to become increasingly important in systems that aggregate file systems. Examples of systems with such support are the FARSITE distributed file-system [Adya et al. 2002], the Pastiche peer-to-peer backup system [Cox et al. 2002], and the single instance store in Windows file servers [Bolosky et al. 2000].

\section{DIRECTORIES}

\subsection{Directory Count per File System}

Figure 13 plots CDFs of file systems by count of directories. The count of directories per file-system has increased steadily over our five-year sample period: The arithmetic mean has grown from 2400 to 8900 directories and the median has grown from $1 \mathrm{~K}$ to $4 \mathrm{~K}$ directories.

We discussed the implications of the rising number of directories per filesystem earlier, in Section 3.1.

\subsection{Directory Size}

This section describes our findings regarding directory size as measured by count of contained files, count of contained subdirectories, and total entry count. None of these size distributions has changed appreciably over our sample period, but the mean count of files per directory has decreased slightly. 


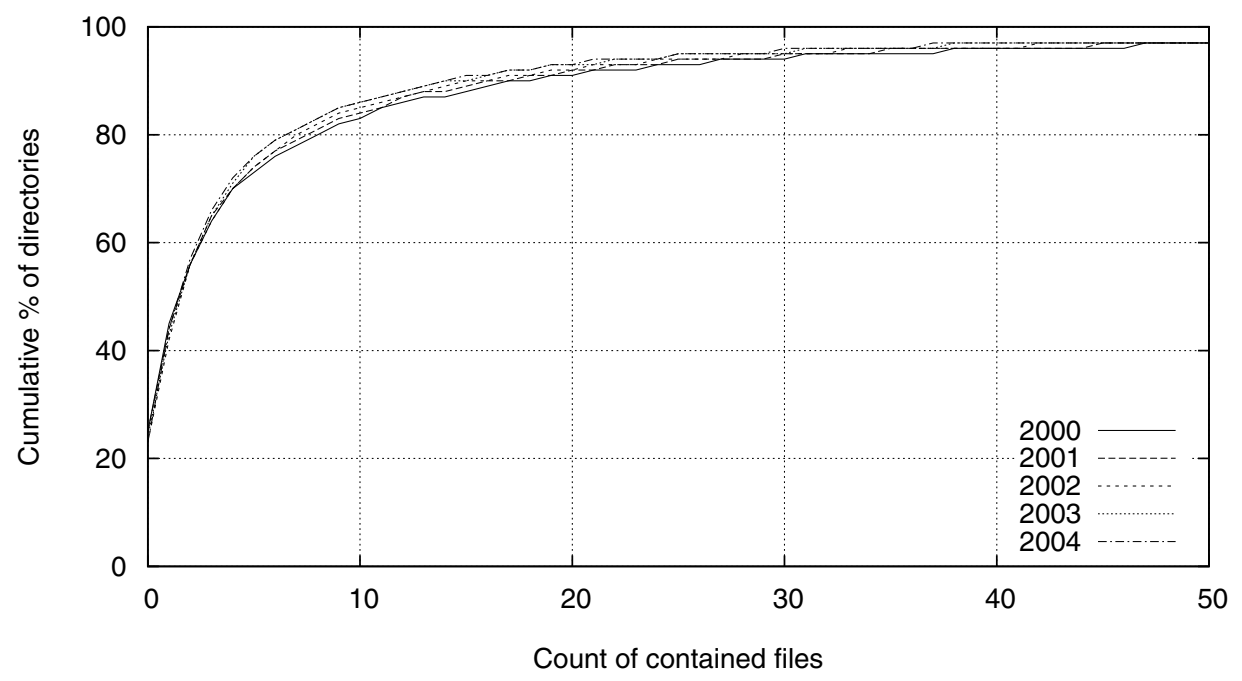

Fig. 14. CDFs of directories by file count.

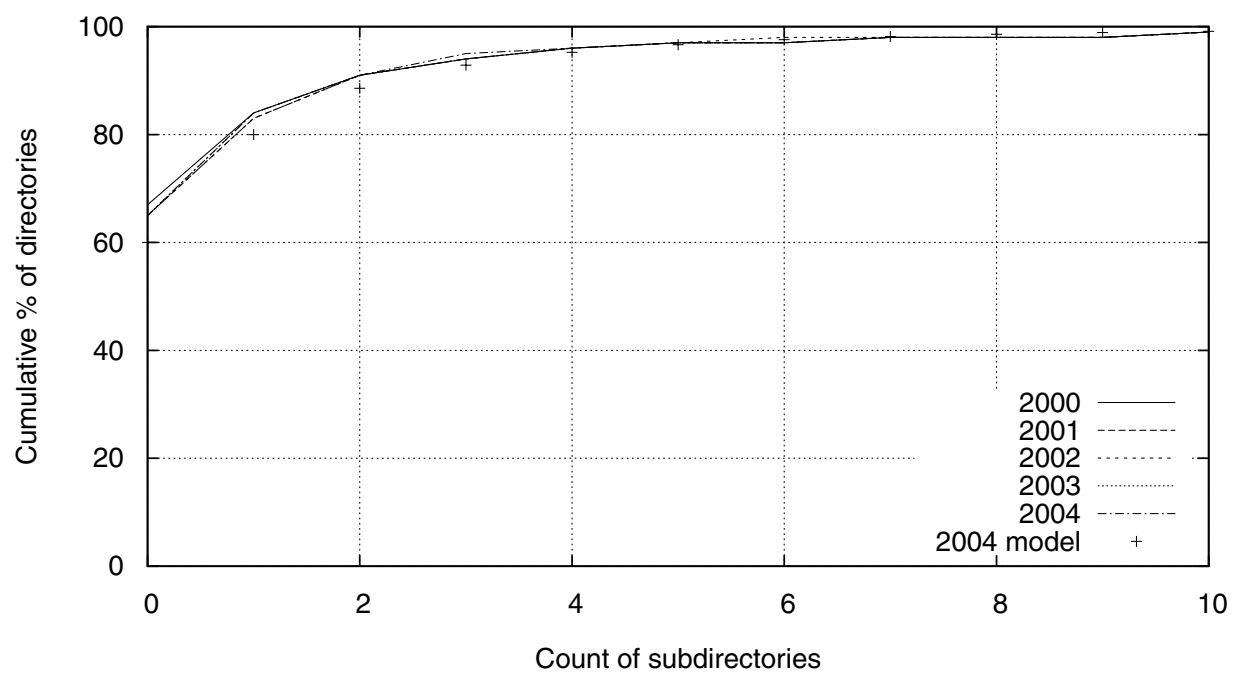

Fig. 15. CDFs of directories by subdirectory count.

Figure 14 plots the CDFs of directories by size, as measured by count of files in the directory. It shows that although the absolute count of directories per file-system has grown significantly over time, the distribution has not changed appreciably. Across all years, $23-25 \%$ of the directories contain no files, which marks a change from 1998 in which only $18 \%$ contained no files and there were more directories containing 1 file than those containing none. The arithmetic mean directory size has decreased slightly and steadily from 12.5 to 10.2 over the sample period, but the median directory size has remained steady at 2 files.

Figure 15 plots the CDFs of directories by size, as measured by count of subdirectories in the directory. It includes a model approximation that we will 


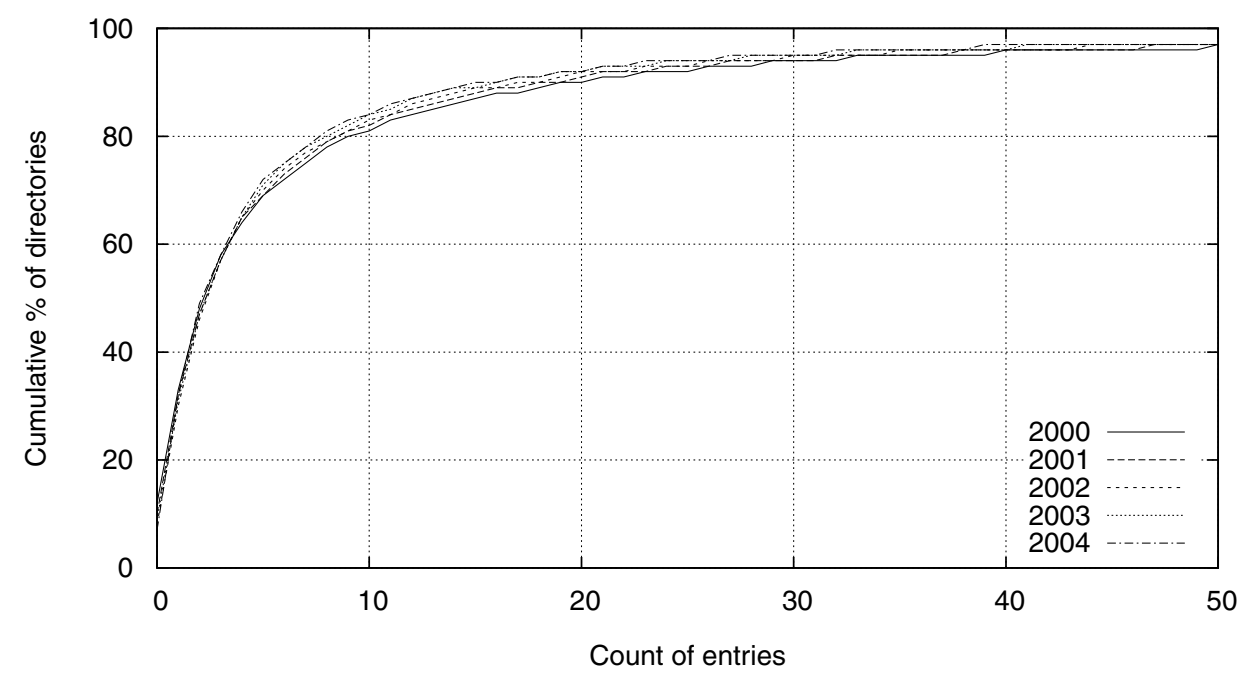

Fig. 16. CDFs of directories by entry count.

discuss later in Section 4.5. This distribution has remained unchanged over our sample period. Across all years, $65-67 \%$ of directories contain no subdirectories, which is similar to the $69 \%$ found in 1998 .

Figure 16 plots CDFs of directories by size, as measured by count of total entries in the directory. This distribution has remained largely unchanged over our sample period. Across all years, 46-49\% of directories contain two or fewer entries.

Since there are so many directories with a small number of files, it would not take much space to colocate the metadata for most of those files with those directories. Such a layout would reduce the seeks associated with file accesses. Therefore, it might be useful to preallocate a small amount of space near a new directory to hold a modest amount of child metadata. Similarly, most directories contain fewer than 20 entries, suggesting using an on-disk structure for directories that optimizes for this common case.

\subsection{Special Directories}

This section describes our findings regarding the usage of Windows special directories. We find that an increasing fraction of file-system storage is in the namespace subtree devoted to system files, and the same holds for the subtree devoted to user documents and settings.

Figure 17 plots the fraction of file-system files that reside within subtrees rooted in each of three special directories: Windows, Program Files, and Documents and Settings. This figure also plots the fraction of file-system bytes contained within each of these special subtrees.

For the Windows subtree, the fractions of files and bytes have both risen from $2-3 \%$ to $11 \%$ over our sample period, suggesting that an increasingly large fraction of file-system storage is devoted to system files. In particular, we note 


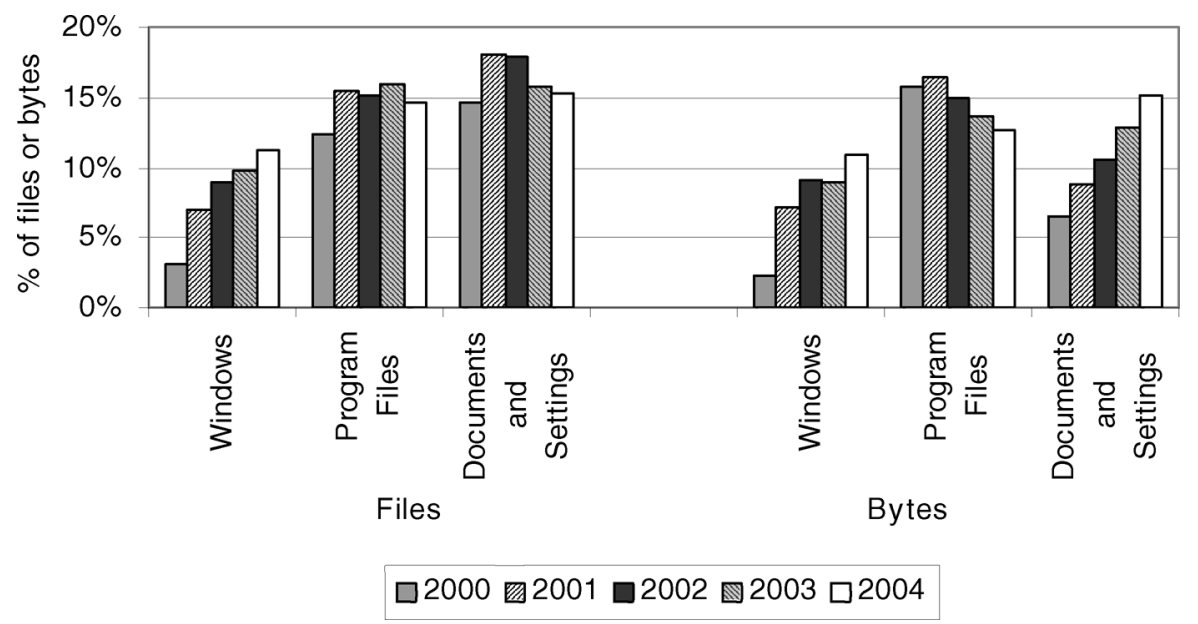

Fig. 17. Fraction of files and bytes in special subtrees.

that Windows XP was released between the times of our 2000 and 2001 data collections.

For the Program Files subtree, the fractions of files and bytes have trended in opposite directions within the range of $12-16 \%$. For the Documents and Settings subtree, the fraction of bytes has increased dramatically while that fraction of files has remained relatively stable.

With regard to the Documents and Settings subtree, the most noticeable trend is that the mean file size used to be half of the mean size overall, but has become roughly the same. This suggests that the nature of files within this subtree has become more consistent with the files overall. We hypothesize that this is because successive versions of Windows have increasingly encouraged placement of user-created files within this subtree [Chapman 2002].

The fraction of all files accounted for by these subtrees has risen from $25 \%$ to $40 \%$, and the fraction that of the bytes therein has risen from $30 \%$ to $41 \%$, suggesting that application writers and end users have increasingly adopted Windows' prescriptive namespace organization [Chapman 2002].

Backup software generally does not have to back-up system files, since they are static and easily restored. Since system files are accounting for a larger and larger fraction of used space, it is becoming increasingly useful for backup software to exclude these files.

On the other hand, files in the Documents and Settings folder tend to be the most important ones to back up, since they contain user-generated content and configuration information. Since the percentage of bytes devoted to these files is increasing, backup capacity planners should expect, surprisingly, that their capacity requirements will increase faster than disk capacity is planned to grow. On the other hand, the percentage of files is not increasing, so they need not expect metadata storage requirements to scale faster than disk capacity. This may be relevant if metadata is backed up in a separate repository from the data, as done by systems such as EMC Centera [Gunawi et al. 2005]. 


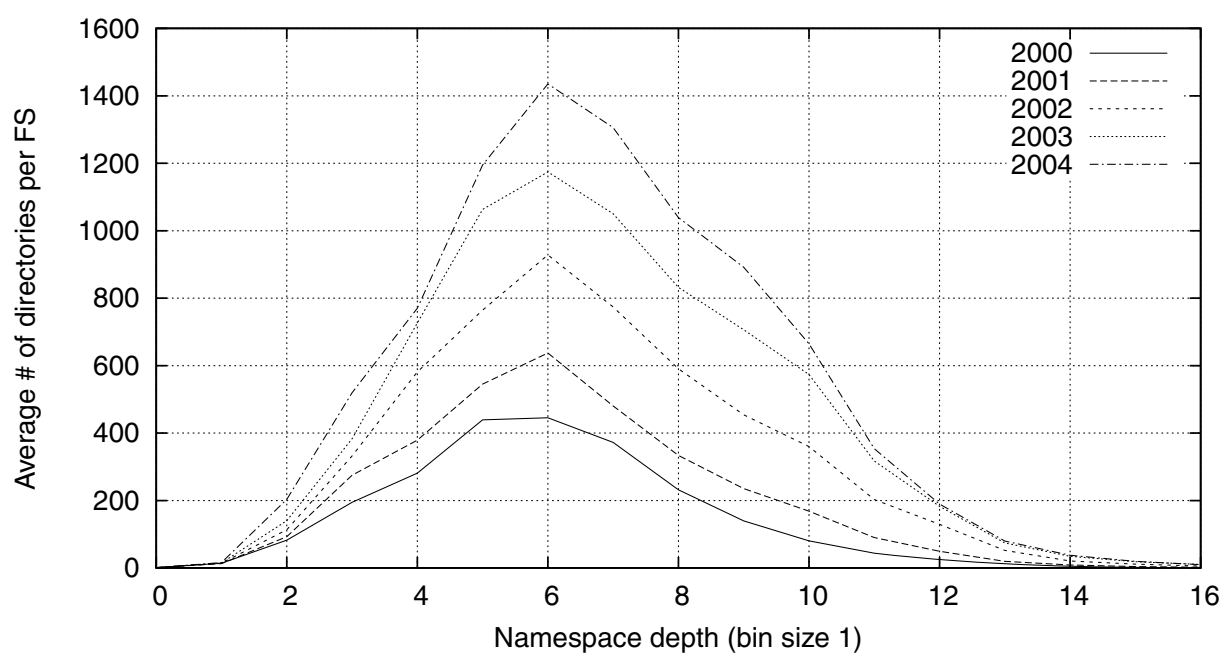

Fig. 18. Histograms of directories by namespace depth.

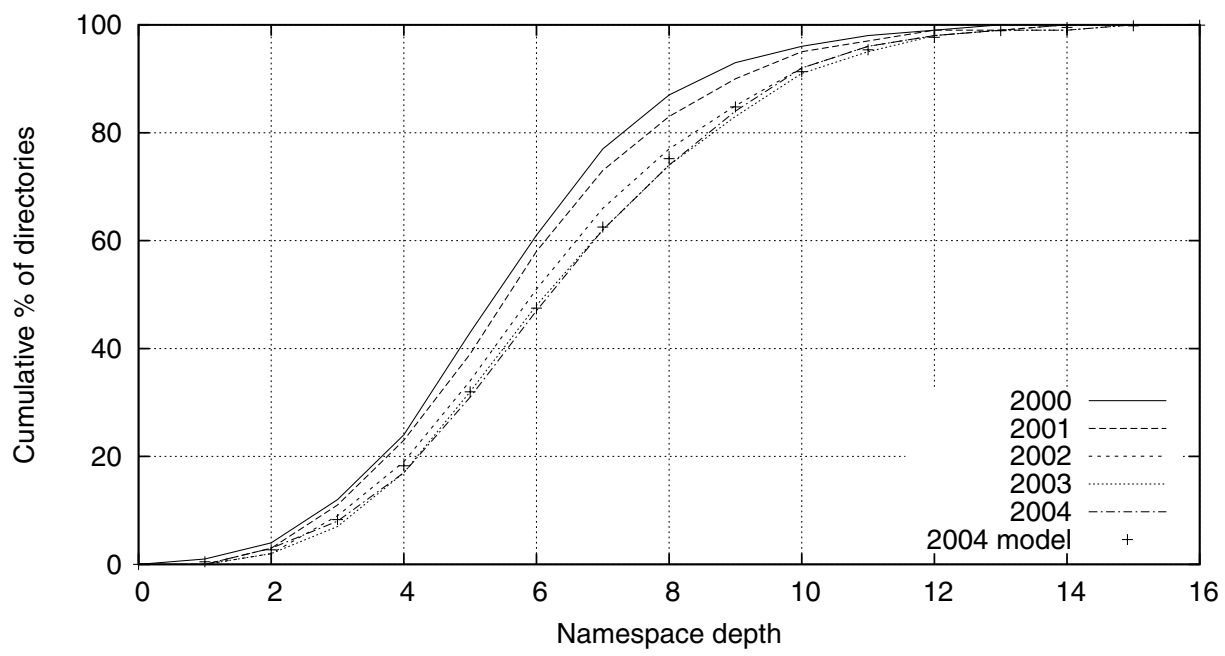

Fig. 19. CDFs of directories by namespace depth.

\subsection{Namespace Tree Depth}

This section describes our findings regarding the depth of directories, files, and bytes in the namespace tree. We find that there are many files deep in the namespace tree, especially at depth 7 . Also, we find that files deeper in the namespace tree tend to be orders of magnitude smaller than shallower files.

Figure 18 plots histograms of directories by their depth in the namespace tree, and Figure 19 shows the CDFs of this same data; it also includes a model approximation we will discuss later in Section 4.5. The general shape of the distribution has remained consistent over our sample period, but the arithmetic 


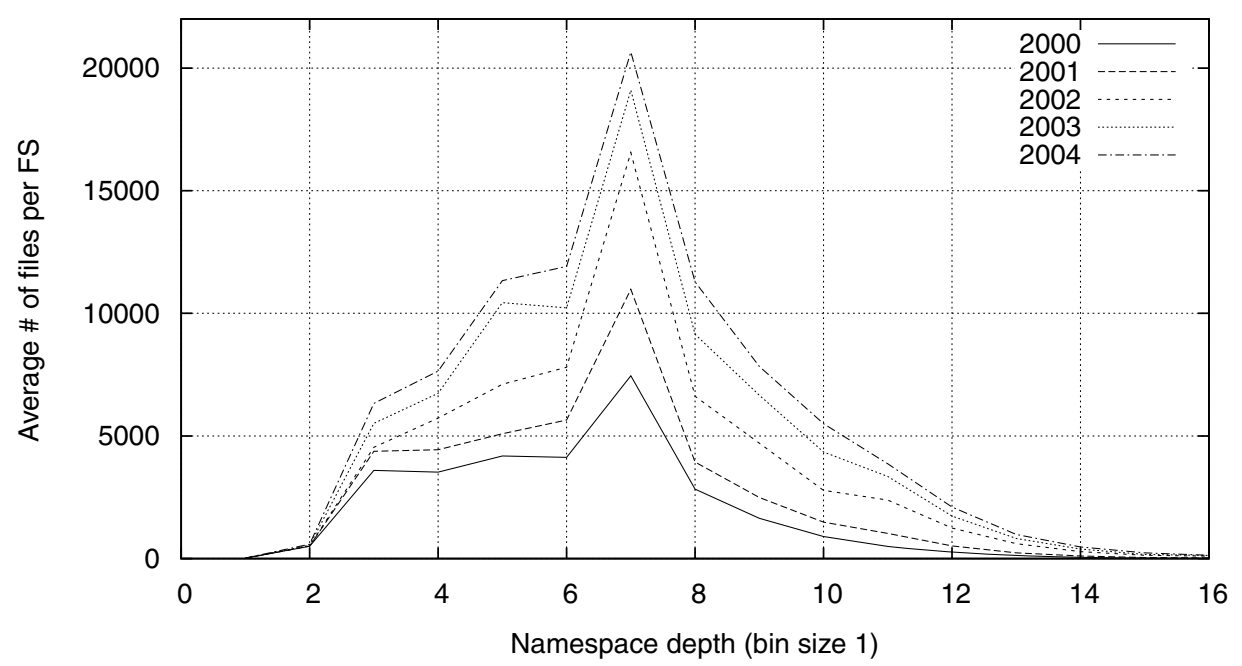

Fig. 20. Histograms of files by namespace depth.

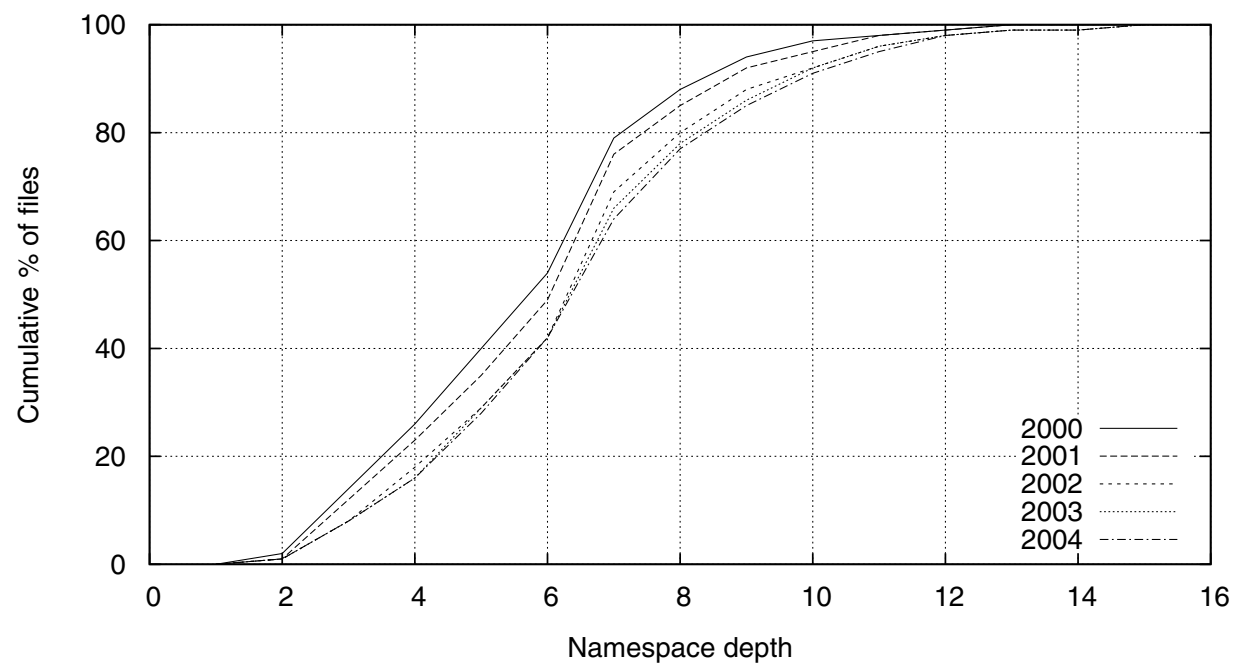

Fig. 21. CDFs of files by namespace depth.

mean has grown from 6.1 to 6.9 , and the median directory depth has increased from 5 to 6 .

Figure 20 plots histograms of file count by depth in the namespace tree, and Figure 21 the CDFs of this same data. With few exceptions, such as at depths 2,3 , and 7 , these distributions roughly track the observed distributions of directory depth, indicating that the count of files per directory is mostly independent of directory depth. To study this more directly, Figure 22 plots the mean count of files per directory versus directory depth. There is a slight downward trend in this ratio with increasing depth, punctuated by three depths whose directories have greater-than-typical counts of files: At depth 2 are files 


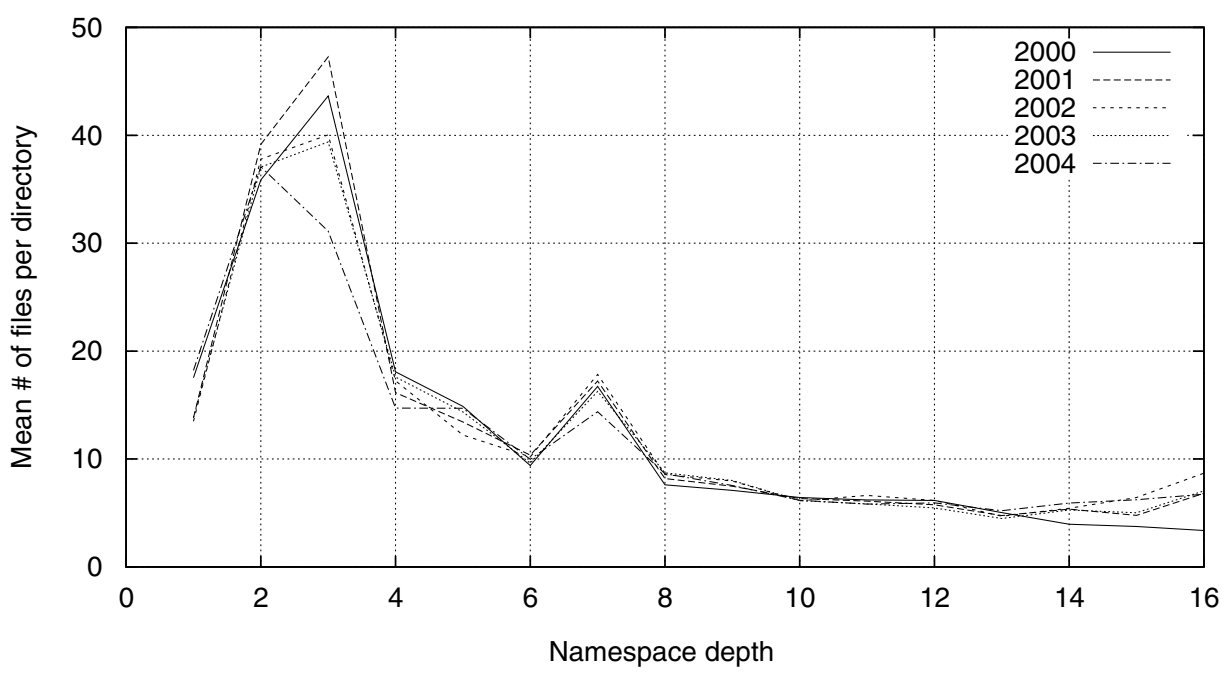

Fig. 22. Files per directory vs. namespace depth.

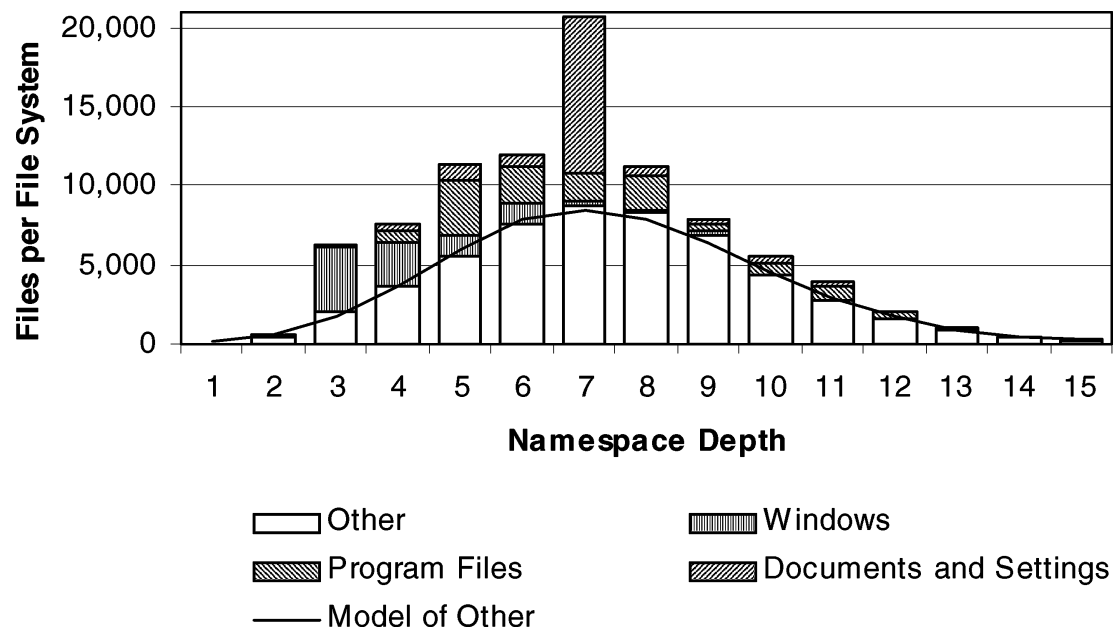

Fig. 23. Contribution of special subtrees to histogram of 2004 files by namespace depth.

in the Windows and Program Files directories; at depth 3 are those in the System and System32 directories; and at depth 7 those in the web cache directories.

Figure 23 replots the 2004 data from Figure 20 as a stacked bar chart, with the indicated contributions of the special namespace subtrees defined in the previous section. Absent these specific types, the distribution of file count by depth in the namespace tree is closely approximated by a Poisson distribution with $\lambda=6.5$, as shown, yielding an MDCC of $1 \%$.

Figure 24 plots histograms of bytes by the depth of their containing files in the namespace tree, and Figure 25 plots CDFs of this same data. These distributions do not closely track the observed distributions of file depth. In particular, files deeper in the namespace tree tend to be smaller than shallower ones. 


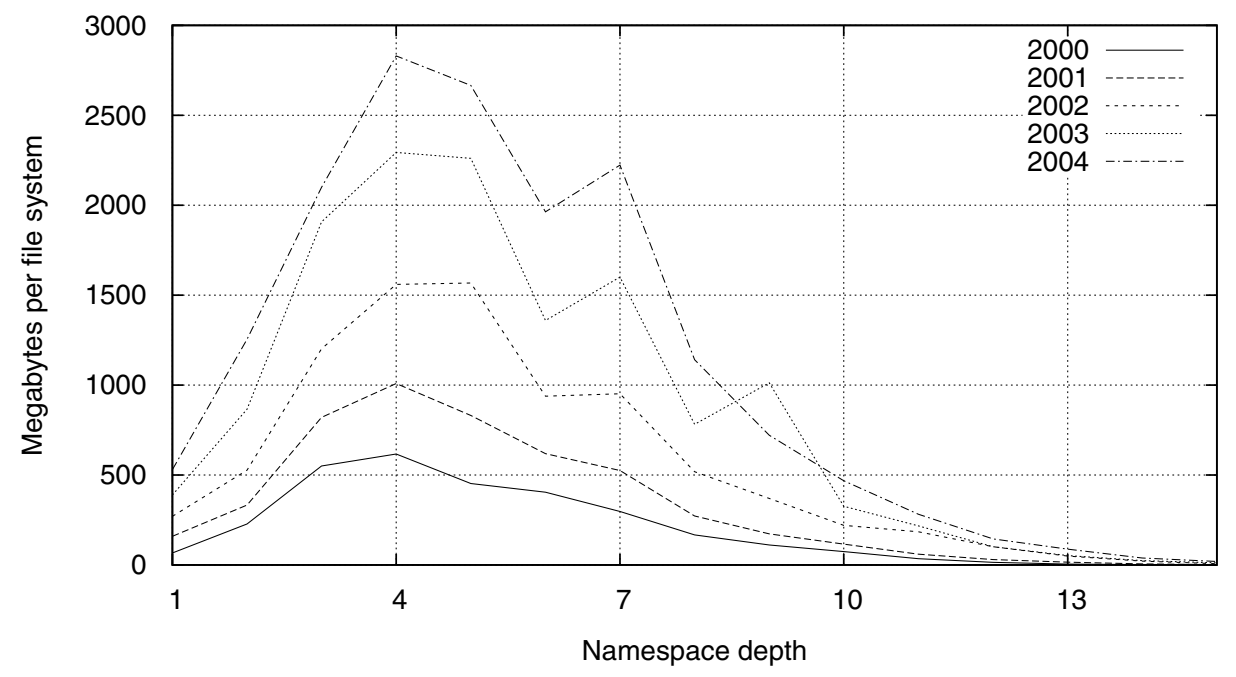

Fig. 24. Histograms of bytes by namespace depth.

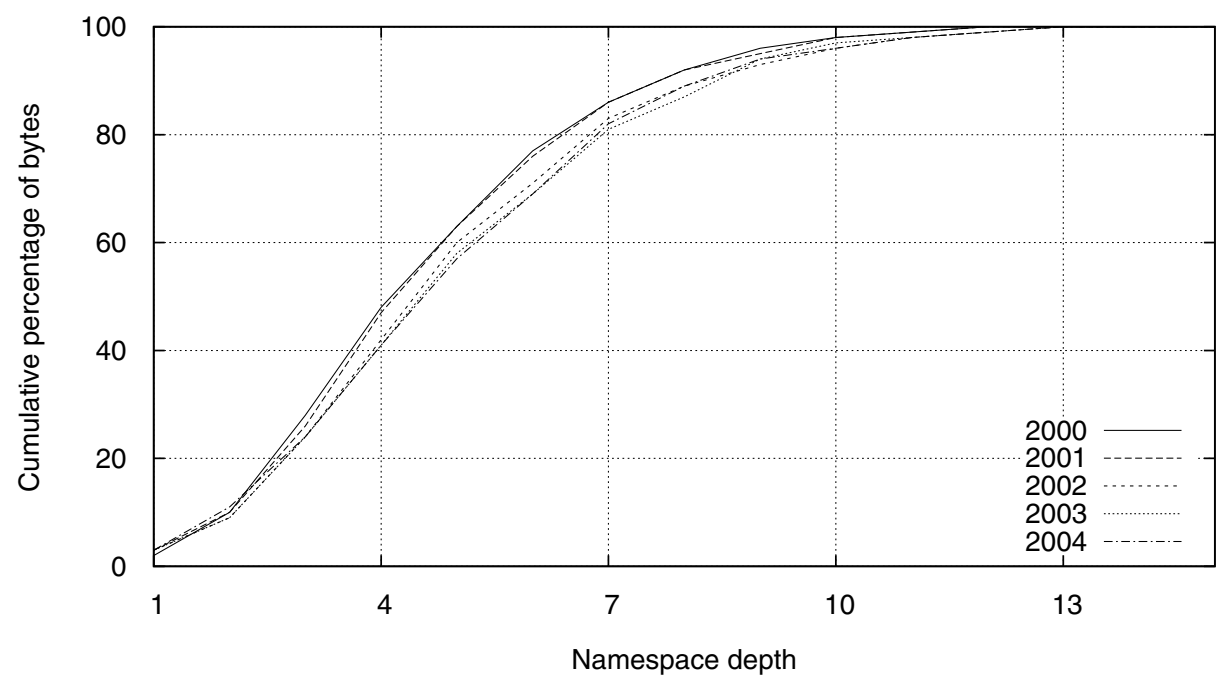

Fig. 25. CDFs of bytes by namespace depth.

This trend is more obvious in Figure 26, which plots the mean file size versus directory depth on a logarithmic scale. We see here that files deeper in the namespace tree tend to be smaller. The mean file size drops by two orders of magnitude between depth 1 and depth 3, and there is a drop of roughly 10\% per depth level thereafter. This phenomenon occurs because most bytes are concentrated in a small number of large files (see Figures 2 and 4), and these files tend to reside in shallow levels of the namespace tree. In particular, the hibernation image file is located in the root.

Since many files and directories are deep in the namespace tree, efficient path lookup of deep paths should be a priority for file-system designers. For 


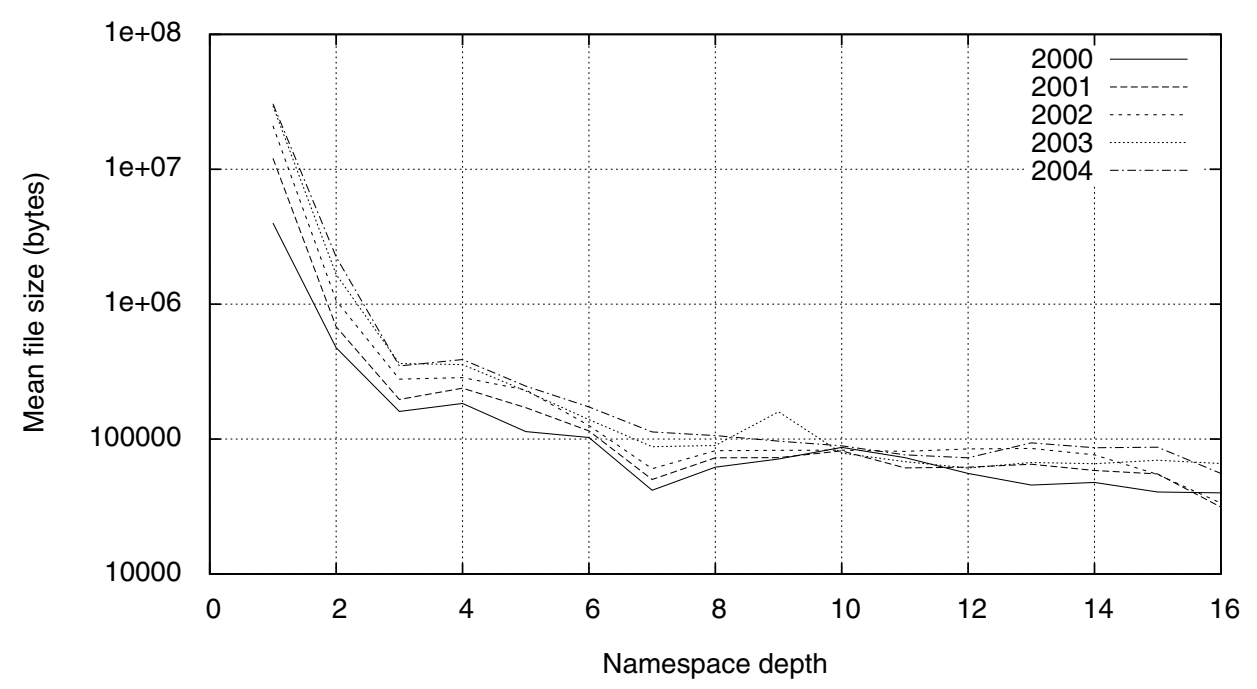

Fig. 26. File size vs. namespace depth.

instance, in distributed file systems where different servers are responsible for various parts of the namespace tree [Adya et al. 2002], deep path lookup may be expensive if not optimized. The high depth of many entries in the namespace may also be of interest to designers of file-system visualization GUIs, to determine how much column space to allot for directory traversal. Furthermore, since the fraction of files at high depths is increasing across the years of our study, these lessons will become more and more important as years pass.

The clear trend of decreasing file size with increasing namespace tree depth sugests a simple coarse mechanism to predict future file size at time of file creation. File systems might use such prediction to decide where on disk to place a new file.

\subsection{Namespace Depth Model}

We have developed a generative model that accounts for the distribution of directory depth. The model posits that new subdirectories are created inside an existing directory in offset proportion to the count of subdirectories already in that directory.

In our previous study [Douceur and Bolosky 1999], we observed that the distribution of directories by depth could be approximated by a Poisson distribution with $\lambda=4.38$, yielding a maximum displacement of cumulative curves (MDCC) of $2 \%$. Poisson is also an acceptable approximation for the five datasets in the present study, with $\lambda$ growing from 6.03 to 6.88 over the sample period, yielding MDCCs that range from $1 \%$ to $4 \%$. However, the Poisson distribution does not provide an explanation for the behavior; it merely provides a means to approximate the result. By contrast, we have developed a generative model that accounts for the distribution of directory depths we have observed, with accuracy comparable to the Poisson model. 
The generative model is as follows. A file-system begins with an empty root directory. Directories are added to the file-system one at-a-time. For each new directory, a parent directory is selected probabilistically, based on the count of subdirectories the parent currently has. Specifically, the probability of choosing each extant directory as a parent is proportional to $c(d)+2$, where $c(d)$ is the count of extant subdirectories of directory $d$. We used Monte Carlo simulation to compute directory depth distributions according to this generative model. Given a count of directories in a file-system, the model produces a distribution of directory depths that matches the observed distribution for file systems of that size. Figure 19 plots the aggregate result of the model for all file systems in the 2004 dataset. The model closely matches the CDF of observed directory depths, with an MDCC of $1 \%$.

Our generative model accounts not only for the distribution of directory depth but also for that of subdirectory size. Figure 15 shows this for the 2004 dataset. The model closely matches the CDF, with an MDCC of $5 \%$.

Intuitively, the proportional probability $c(d)+2$ can be interpreted as follows: If a directory already has some subdirectories, it has demonstrated that it is a useful location for subdirectories, and so is a likely place for more subdirectories to be created. The more subdirectories it has, the more demonstrably useful it has been as a subdirectory home, so the more likely it is to continue to spawn new subdirectories. If the probability were proportional to $c(d)$ without any offset, then an empty directory could never become nonempty, so some offset is necessary. We found an offset of 2 to match our observed distributions very closely for all five years of our collected data, but we do not understand why the particular value of 2 should be appropriate.

\section{SPACE USAGE}

\subsection{Capacity and Usage}

Figure 27 plots CDFs of file-system volumes by storage capacity, which has increased dramatically over our five-year sample period: The arithmetic mean has grown from $8 \mathrm{~GB}$ to $46 \mathrm{~GB}$ and the median has grown from $5 \mathrm{~GB}$ to $40 \mathrm{~GB}$. The number of small-capacity file-system volumes has dropped dramatically: Systems of $4 \mathrm{~GB}$ or less have gone from $43 \%$ to $4 \%$ of all file systems.

Figure 28 plots CDFs of file systems by total consumed space, including not only file content but also space consumed by internal fragmentation, file metadata, and the system paging file. Space consumption increased steadily over our five-year sample period: The geometric mean from $1 \mathrm{~GB}$ to $9 \mathrm{~GB}$, the arithmetic mean from $3 \mathrm{~GB}$ to $18 \mathrm{~GB}$, and the median from $2 \mathrm{~GB}$ to $13 \mathrm{~GB}$.

Figure 29 plots CDFs of file systems by percentage of fullness, meaning the consumed space relative to capacity. The distribution is very nearly uniform for all years, as it was in our 1998 study. The mean fullness has dropped slightly from $49 \%$ to $45 \%$, and the median file-system has gone from $47 \%$ full to $42 \%$ full. By contrast, the aggregate fullness of our sample population, computed as total consumed space divided by total file-system capacity, has held steady at $41 \%$ over all years. 


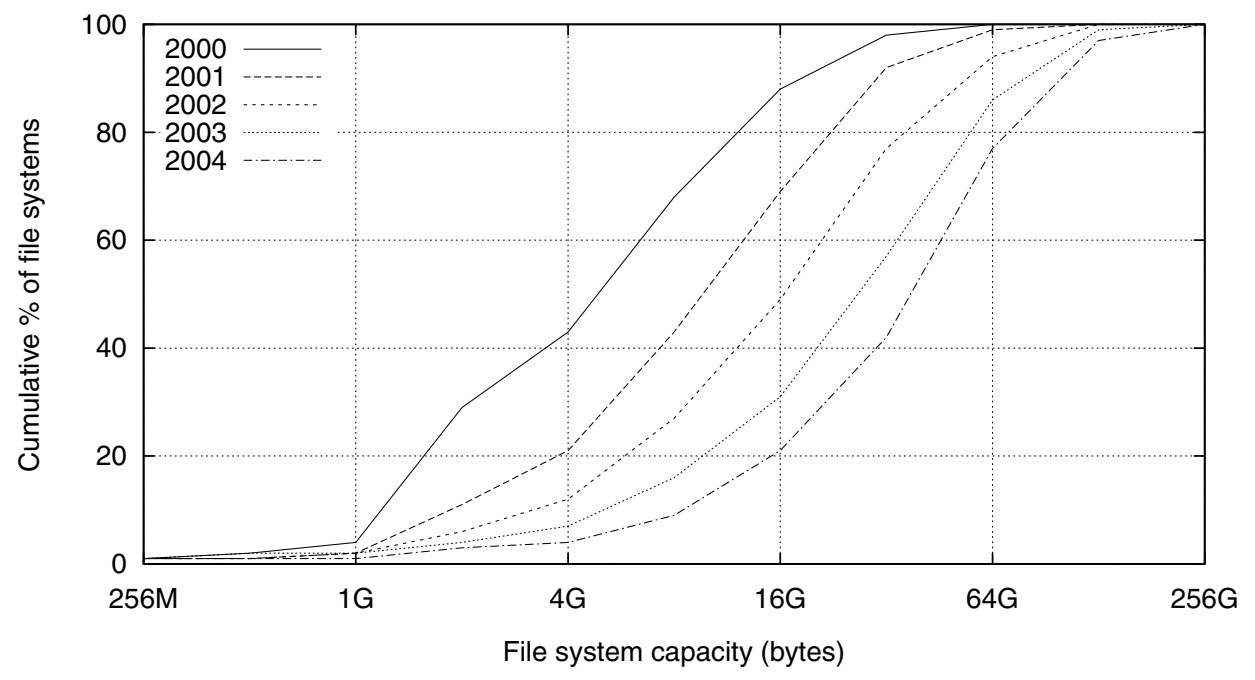

Fig. 27. CDFs of file systems by storage capacity.

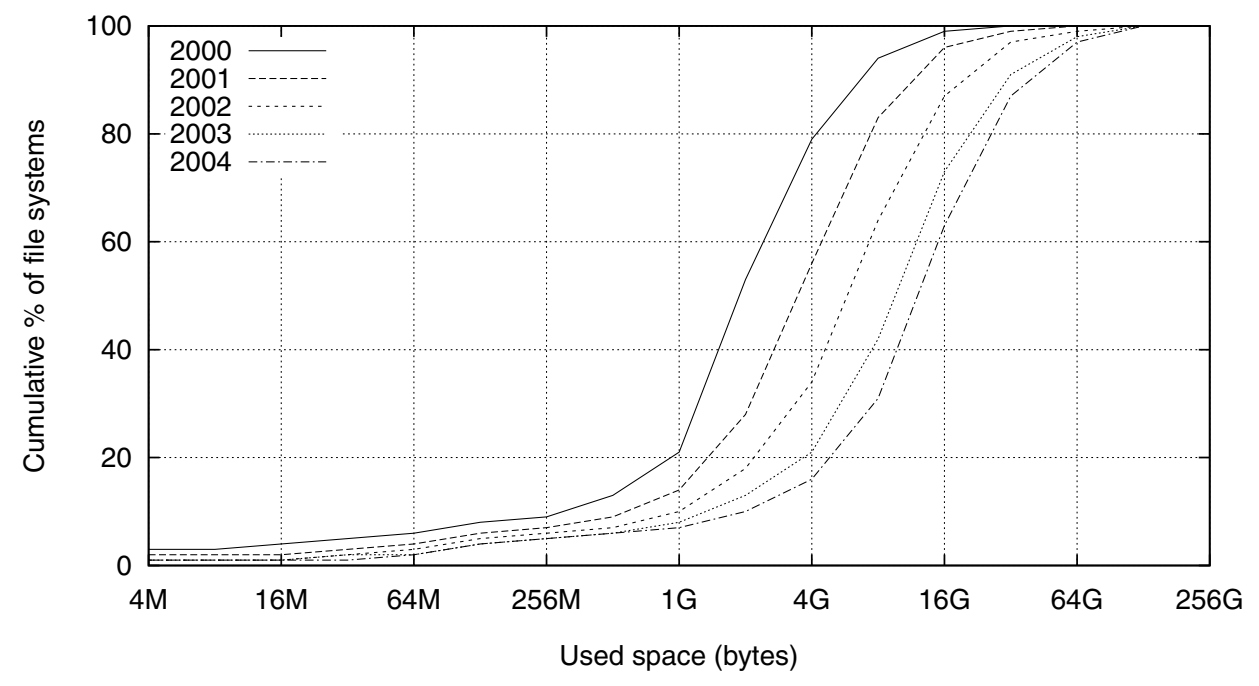

Fig. 28. CDFs of file systems by total consumed space.

In any given year, the range of file-system capacities in this organization is quite large. This means that software must be able to accommodate a wide range of capacities simultaneously existing within an organization. For instance, a peer-to-peer backup system must be aware that some machines will have drastically more capacity than others. File system designs, which must last many years, must accommodate even more dramatic capacity differentials.

\subsection{Changes in Usage}

This subsection describes our findings regarding how individual file systems change in fullness over time. For this part of our work, we examined the 6536 


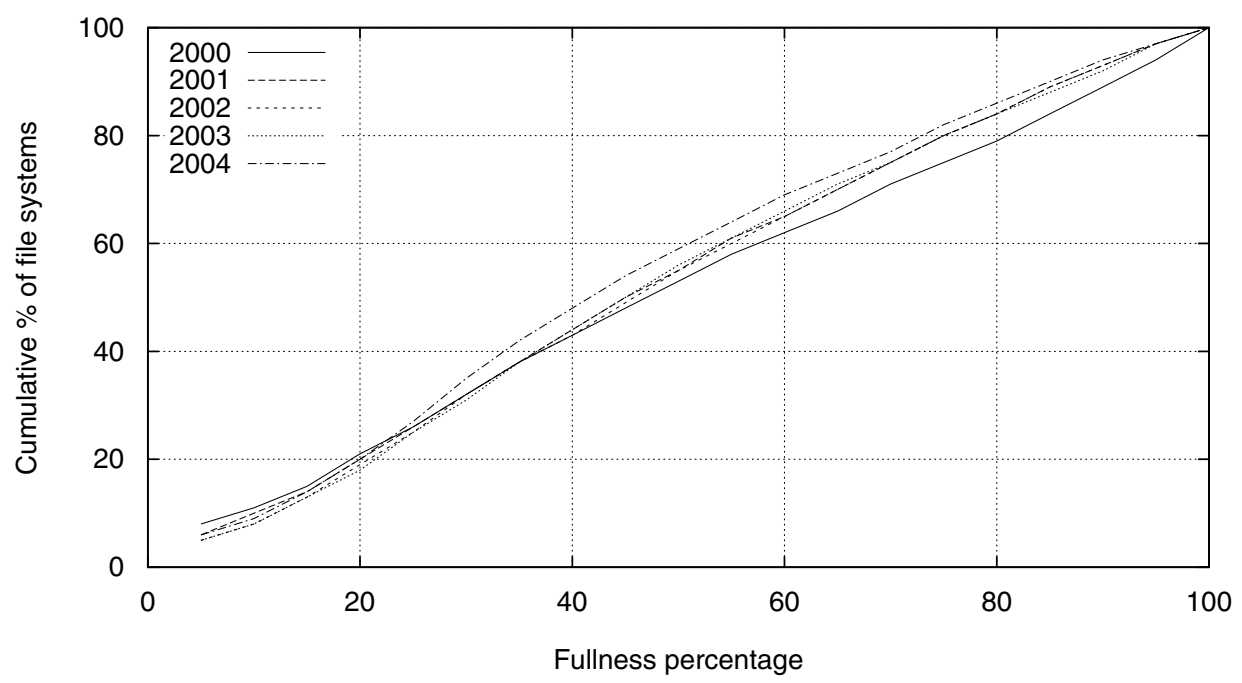

Fig. 29. CDFs of file systems by fullness.

snapshot pairs that correspond to the same file-system in two consecutive years. We also examined the 1320 snapshot pairs that correspond to the same filesystem two years apart. We find that $80 \%$ of file systems become fuller over a one-year period, and the mean increase in fullness is 14 percentage points. This increase is predominantly due to creation of new files, partly offset by deletion of old files rather than due to extant files changing size.

When comparing two matching snapshots in different years, we must establish whether two files in successive snapshots of the same file-system are the same file. We do not have access to files' inode numbers because collecting them would have lengthened our scan times to an unacceptable degree. We thus instead use the following proxy for file sameness: If the files have the same full pathname, they are considered the same, otherwise they are not. This is a conservative approach: It will judge a file to be two distinct files if it or any ancestor directory has been renamed.

Figures 30 and 31 plot histograms and CDFs, respectively, of file systems by percentage-point increase in fullness from one year to the next. We define this term by example: If a file-system was 50\% full in 2000 and $60 \%$ full in 2001 , it exhibited a 10 percentage-point increase in fullness. The distribution is substantially the same for all four pairs of consecutive years. Figure 31 shows that $80 \%$ of file systems exhibit an increase in fullness and fewer than $20 \%$ show a decrease. The mean increase from one year to the next is 14 percentage points.

We also examined the increase in fullness over two years. We found the mean increase to be 22 percentage points. This is less than twice the consecutiveyear increase, indicating that as file systems age, they increase their fullness at a slower rate. Because we have so few file systems with snapshots in four consecutive years, we did not explore increases over three or more years.

Since file systems that persist for a year tend to increase their fullness by about 14 points, but the mean file-system fullness has dropped from $49 \%$ to 


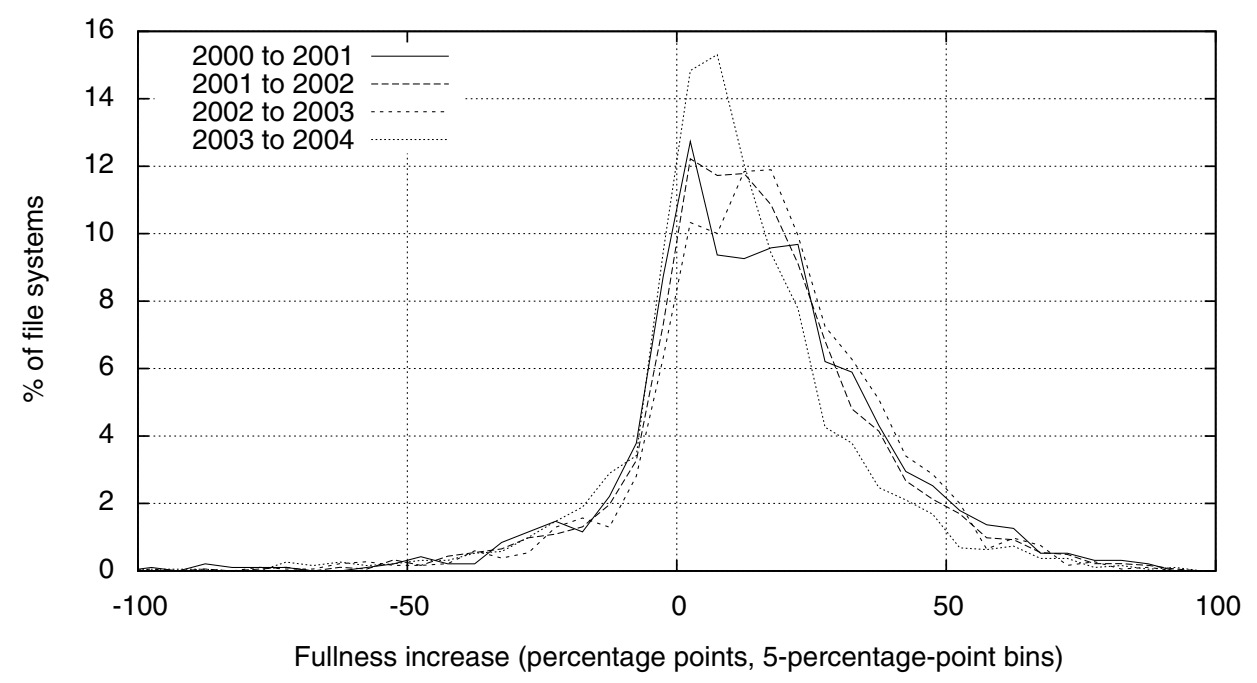

Fig. 30. Histograms of file systems by 1-year fullness increase.

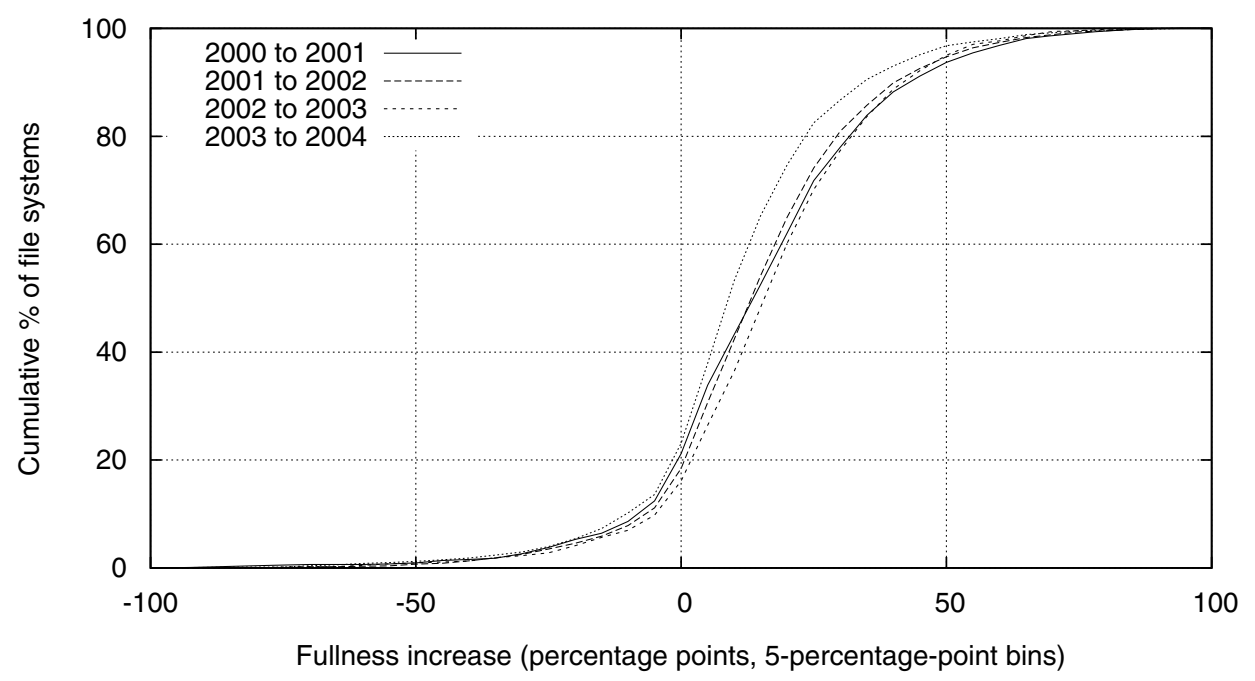

Fig. 31. CDFs of file systems by 1-year fullness increase.

$45 \%$ over our sample period, it seems that the steadily increasing fullness of individual file systems is offset by the replacement of old file systems with newer, emptier ones.

Analyzing factors that contribute to the 14-point mean year-to-year increase in fullness revealed the following breakdown: Fullness increases by 28 percentage points due to files that are present in the later snapshot but not in the earlier one, meaning that they were created during the intervening year. Fullness decreases by 15 percentage points due to files that are present in the earlier snapshot but not in the later one, meaning that they were deleted during the intervening year. Fullness also increases by 1 percentage point due 
to growth in the size of files that are present in both snapshots. An insignificant fraction of this increase is attributable to changes in system paging files, internal fragmentation, or metadata storage.

We examined the size distributions of files that were created and of those that were deleted, to see if they differed from the overall file-size distribution. We found that they do not differ appreciably. We had hypothesized that users tend to delete large files to make room for new content, but the evidence does not support this hypothesis.

Since deleted and created files have similar size distributions, file-system designers need not expect the fraction of files of different sizes to change as a file-system ages. Thus, if they find it useful to assign different parts of the disk to files of different sizes, they can anticipate that the allocation of sizes to disk areas will not need radical change as time passes.

Many peer-to-peer systems use free space on computers to store shared data, so the amount of used space is of great importance. With an understanding of how this free space decreases as a file-system ages, a peer-to-peer system can proactively plan how much it will need to offload shared data from each file-system to make room for additional local content. Also, since a common reason for upgrading a computer is because its disk space becomes exhausted, a peer-to-peer system can use a prediction of when a file-system will become full as a coarse approximation to when that file-system will become unavailable.

\section{RELATED WORK}

This research extends our earlier work in measuring and modeling file-system metadata on Windows workstations. In 1998, we collected snapshots of over 10,000 file systems on the desktop computers at Microsoft [Douceur and Bolosky 1999]. The focus of the earlier study was on variations among file systems within the sample, all of which were captured at the same time. By contrast, the focus of the present study is on longitudinal analysis, meaning how file systems have changed over time.

Prior to our previous study, there were no studies of static file-system metadata on Windows systems, but there were several such studies in other operating system environments. These include Satyanarayanan's study of a digital PDP-10 at CMU [Satyanarayanan 1981], Mullender and Tanenbaum's study of a Unix system at Vrije Universiteit [Mullender and Tanenbaum 1984], Irlam's study of 1050 Unix file systems [Irlam 1993], and Sienknecht et al.'s study of 267 file systems in 46 HP-UX systems at Hewlett-Packard [Sienknecht et al. 1994]. All of these studies involved snapshots taken at a single time, like our study in 1998. There have also been longitudinal studies of file-system metadata, but for significantly shorter times than ours: Bennett et al. studied three file servers at the University of Western Ontario over a period of one day in 1991 [Bennett et al. 1991], and Smith and Seltzer studied 48 file systems on four file servers at Harvard over a period of ten months [Smith and Seltzer 1994].

We are aware of only one additional collection of static file-system metadata since our previous study. In 2001, Evans and Kuenning captured snapshots 
from 22 machines running various operating systems at Harvey Mudd College and Marine Biological Laboratories [Evans and Kuenning 2002]. Their data collection and analysis focused mainly, but not exclusively, on media files. Their findings show that different types of files exhibit significantly disparate size distributions, which our results support.

Many studies have examined dynamic file-system traces rather than static file-system snapshots. These studies are complementary to ours, describing things we cannot analyze, such as the rate at which bytes are read and written in a file-system. A few examples of such studies are Ousterhout et al.'s analysis of the BSD file-system [Ousterhout et al. 1985], Gribble et al.'s analysis of selfsimilarity in the dynamic behavior of various file systems [Gribble et al. 1998], Vogels's analysis of Windows NT [Vogels 1999], and Roselli et al.'s analysis of HP-UX and Windows NT [Roselli et al. 2000].

In addition to file-system measurement research, there has been much work in modeling file-system characteristics, most notably those related to the distribution of file sizes. Examples of work in this area include that of Satyanarayanan [1981], Barford and Crovella [1998], Downey [2001], and Mitzenmacher [2004].

In 2001, Evans and Kuenning broke down measured file-size distributions according to file type, and modeled the sizes using log-lambda distributions [Evans and Kuenning 2002]. They found that video and audio files can significantly perturb the file-size distribution and prevent simple size models from applying. We did not find this to be true for file sizes in our sample population. However, we did find video, database, and blob files responsible for a second peak in the distribution of bytes by containing file size.

In our previous study, we modeled directory depth with a Poisson distribution [Douceur and Bolosky 1999], but we have herein proposed a generative model in which the attractiveness of an extant directory $d$ as a location for a new subdirectory is proportional to $c(d)+2$, where $c(d)$ is the count of directory $d$ 's extant subdirectories. This is strikingly similar to the rule for generating plane-oriented recursive trees, wherein the probability is proportional to $c(d)+1$ [Mahmoud 1992].

\section{SUMMARY AND CONCLUSIONS}

Over a span of five years, we collected metadata snapshots from more than 63,000 distinct Windows file systems in a commercial environment, through voluntary participation of the systems' users. These systems contain 4 billion files totaling 700TB of file data. For more than $10 \%$ of these file systems, we obtained snapshots in multiple years, enabling us to directly observe how these file systems have changed over time. Our measurements reveal several interesting properties of file systems and offer useful lessons.

One interesting discovery is the emergence of a second mode in the GB range in the distribution of bytes by containing file size. It makes us wonder whether at some future time a third mode will arise. The increasingly large fraction of content in large files suggests that variable block sizes, as supported by ZFS [Bonwick 2006] and NTFS [Solomon 1998], are becoming increasingly 
important. Since a few large files, mainly video, database, and blob, are contributing to an increasing fraction of file-system usage, these file extensions are ideal candidates for larger block sizes.

Although large files account for a large fraction of space, most are $4 \mathrm{~KB}$ or smaller. Thus, it is useful to colocate several small files in a single block, as ReiserFS [Reiser 2006] does, and to colocate small file content with file metadata, as NTFS does. Our finding that most directories have few entries suggests yet another possibility: Colocate small file content with the file's parent directory. An even more extreme solution is suggested by the fact that in 2004, the average file-system had only $52 \mathrm{MB}$ in files $4 \mathrm{~KB}$ or smaller. Since this number is becoming small relative to main memory sizes, it may soon be practical to avoid cache misses entirely for small files by prefetching them all at boot time and pinning them in the cache.

Another noteworthy discovery is that the fraction of files locally modified decreases with time, an effect significant enough to be observable in only a five-year sample. It would appear that users' ability to generate increasing amounts of content is outstripped by the phenomenal growth in their disks. If the practice of individuals copying content from each other becomes increasingly common, then applications like peer-to-peer backup will have increasing amounts of intermachine content similarity to leverage so as to obviate copying.

We were surprised to find a strong negative correlation between namespace depth and file size. Such a strong and temporally-invariant correlation, in combination with the well-known one between file extension and file size, can help us make predictions of file size at creation time. This may be useful, for example, in deciding how many blocks to initially allocate to a file.

We also discovered that a simple generative model can account for both distributions of directory depth and the count of subdirectories per directory. The model we developed posits that new subdirectories are created inside an existing directory in offset proportion to the count of subdirectories already in that directory. This behavior is easy to simulate, and produces directory-depth and directory-size distributions that closely match our observations.

Finally, it is remarkable that file-system fullness over the course of five years has changed little, despite the vast increase in file-system capacity over that same period. It seems clear that users scale their capacity needs to their available capacity. The lesson for storage manufacturers is to keep focusing effort on increasing capacity because customers will continue to place great value on this aspect for the foreseeable future.

We have made our traces available to the community via the Storage Networking Industry Association's IOTTA repository. To obtain them, visit the URL http://iotta.snia.org/traces/tracesStaticSnapshot/.

\section{ACKNOWLEDGMENTS}

The authors would like to express great thanks to the many Microsoft users who consented to us taking snapshots of their file systems. Without them, this study would have been impossible. We also thank Jim Gray, Catharine van Ingen, Tim Harris, and Randal Burns for their comments on early drafts of 
this work. Finally, we thank the Storage Networking Industry Association for making our traces available to the public via their IOTTA repository.

\section{REFERENCES}

Adya, A., Bolosky, W., Castro, M., Cermak, G., Chaiken, R., Douceur, J., Howell, J., Lorch, J., TheImer, M., AND WATtenhofer, R.P. 2002. FARSITE: Federated, available, and reliable storage for an incompletely trusted environment. In Proceedings of the 5th USENIX Symposium on Operating Systems Design and Implementation (OSDI), Boston, MA, 1-14.

Agrawal, N.A., Bolosky, W.J., Douceur, J.R., And Lorch, J.R. 2007. A five-year study of file system metadata. In Proceedings of the 5th USENIX Conference on File and Storage Technologies (FAST), San Jose, CA, 31-45.

Arpaci-Dusseau, A.C. AND Arpaci-Dusseau, R.H. 2001. Information and control in gray-box systems. In Proceedings of the 18th ACM Symposium on Operating Systems Principles (SOSP), Banff, Canada, 43-56.

BARford, P. ANd Crovella, M. 1998. Generating representative web workloads for network and server performance evaluation. In Proceedings of the Joint International Conference on Measurement and Modeling of Computer Systems (SIGMETRICS), Madison, WI, 151-160.

Bennett, J.M., Bauer, M.A., And Kinchlea, D. 1991. Characteristics of files in NFS environments. In Proceedings of the ACM SIGSMALL/PC Symposium on Small Systems, Toronto, Ontario, Candada, 33-40.

Bolosky, W.J., Corbin, S., Goebel, D., And Douceur, J.R. 2000. Single instance storage in Windows 2000. In Proceedings of the 4th USENIX Windows Systems Symposium, Seattle, WA.

Bonwick, J. 2006. ZFS: The last word in file systems. http://www.opensolaris.org/os/community/ zfs/docs/zfs_last.pdf.

Chapman, G. 2002. Why does Explorer think I only want to see my documents? http:// pubs.logicalexpressions.com/Pub0009/LPMArticle.asp?ID=189.

Cox, L.P., Murray, C.D., And Noble, B.D. 2002. Pastiche: Making backup cheap and easy. In Proceedings of the 5th USENIX Symposium on Operating Systems Design and Implementation (OSDI), Boston, MA, 285-298.

Douceur, J.R. AND Bolosky, W.J. 1999. A large-scale study of file system contents. In Proceedings of the Joint International Conference on Measurement and Modeling of Computer Systems (SIGMETRICS), Atlanta, GA, 59-70.

Downey, A.B. 2001. The structural cause of file size distributions. In Proceedings of the Joint International Conference on Measurement and Modeling of Computer Systems (SIGMETRICS), Cambridge, MA, 328-329.

Evans, K.M. and Kuenning, G.H. 2002. A study of irregularities in file-size distributions. In Proceedings of the International Symposium on Performance Evaluation of Computer and Telecommunication Systems (SPECTS), San Diego, CA.

Freund, J.E. 1992. Mathematical Statistics, 5th ed. Prentice Hall.

Gribble, S.D., Manku, G.S., Roselli, D.S., Brewer, E.A., Gibson, T.J., and Miller, E.L. 1998. SelfSimilarity in file systems. In Proceedings of the Joint International Conference on Measurement and Modeling of Computer Systems (SIGMETRICS), Madison, WI, 141-150.

Gunawi, H.S., Agrawal, N., Arpaci-Dusseau, A.C., Arpaci-Dusseau, R.H., and Schindler, J. 2005. Deconstructing commodity storage clusters. In Proceedings of the 32 nd International Symposium on Computer Architecture (ISCA), Madison, WI, 60-71.

IRLAM, G. 1993. Unix file size survey - 1993. http://www.base.com/gordoni/ufs93.html.

Knuth, D.E. 1981. The Art of Computer Programming, Volume 2: Seminumerical Algorithms, 2nd ed. Addison-Wesley.

Mahmoud, H.M. 1992. Distances in random plane-oriented recursive trees. J. Comput. Appl. Math. 41, 237-245.

Mesnier, M., Thereska, E., Ganger, G.R., Ellard, D., and Seltzer, M. 2004. File classification in self-* storage systems. In Proceedings of the 1st International Conference on Autonomic Computing $(I C A C)$, New York.

Microsoft. 2006. SetFileTime. http://msdn.microsoft.com/library/default.asp?url=/library/enus/wcecoreos $5 / \mathrm{html} /$ wce50lrfsetfiletime.asp. 
Mitchell, S. 1997. Inside the Windows 95 file system. O’Reilly, Sebastopol, CA.

Mitzenmacher, M. 2004. Dynamic models for file sizes and double Pareto distributions. Internet Math. 1, 3, 305-333.

Mullender, S.J. and Tanenbaum, A.S. 1984. Immediate files. Softw. Pract. Exper. 14, 4 (Apr.), 365-368.

Ousterhout, J.K., Costa, H.D., Harrison, D., Kunze, J.A., Kupfer, M., And Thompson, J.G. 1985. A trace-driven analysis of the UNIX 4.2 BSD file system. In Proceedings of the 10th ACM Symposium on Operating Systems Principles (SOSP), Orcas Island, WA, 15-24.

REISER, H. 2006. Three reasons why ReiserFS is great for you. http://www.namesys. com/.

RoselLi, D., Lorch, J.R., AND Anderson, T.E. 2000. A comparison of file system workloads. In Proceedings of the USENIX Annual Technical Conference, San Diego, CA, 41-54.

Satyanarayanan, M. 1981. A study of file sizes and functional lifetimes. In Proceedings of the 8th ACM Symposium on Operating Systems Principles (SOSP), Pacific Grove, CA, 96-108.

Sienknecht, T.F., Friedrich, R.J., Martinka, J.J., and Friedendach, P.M. 1994. The implications of distributed data in a commercial environment on the design of hierarchical storage management. In Proceedings of the 16th IFIP Working Group 7.3 International Symposium on Computer Performance Modeling and Evaluation. 3-25.

Smith, K. And Seltzer, M. 1994. File layout and file system performance. Tech. Rep. TR-35-94, Harvard University.

Solomon, D.A. 1998. Inside Windows NT, 2nd ed. Microsoft Press, Redmond, WA.

Vogels, W. 1999. File system usage in Windows NT 4.0. In Proceedings of the 17th ACM Symposium on Operating Systems Principles (SOSP), Kiawah Island, SC, 93-109.

Received August 2007; accepted August 2007 